\title{
Manuscript Details
}

\section{Manuscript number}

Title
IJBIOMAC_2018_6721_R1

Chitosan coated human serum albumin nanoparticles: a promising strategy for nose-to-brain drug delivery.

Research Paper

\section{Article type}

Abstract

The aim of the present study was the development of human serum albumin nanoparticles (HSA NPs) as nose-tobrain carrier. To strengthen, the efficacy of nanoparticles as drug delivery system, the influence of chitosan (CS) coating on the performance of HSA NPs was investigated for nasal application. HSA NPs were prepared by desolvation technique. CS coating was obtained adding the CS solution to HSA NPs. The mean particle size was $241 \square 18 \mathrm{~nm}$ and $261 \square 8 \mathrm{~nm}$ and the $\square$-potential was $-47 \square 3 \mathrm{mV}$ and $+45 \square 1 \mathrm{mV}$ for HSA NPs and CS-HSA NPs, respectively. The optimized formulations showed excellent stability upon storage both as suspension and as freezedried product after 3 months. The mucoadhesion properties were assessed by turbidimetric and indirect method. NPs were loaded with sulforhodamine B sodium salt as model drug and the effect of CS coating was investigated performing release studies, permeation and uptake experiments using Caco-2 and hCMEC/D3 cells as model of the nasal epithelium and blood-brain barrier, respectively. Furthermore, ex vivo diffusion experiments have been carried out using rabbit nasal mucosa. Finally, the ability of the formulations to reversibly open tight and gap junctions was explored by western blotting and RT-PCR analyzing in both Caco-2 and hCMEC/D3 cells.

\section{Keywords}

Manuscript category

Corresponding Author

Corresponding Author's Institution

\section{Order of Authors}

Suggested reviewers

\author{
Albumin nanoparticles; Chitosan; nose to brain. \\ Carbohydrates, Natural Polyacids and Lignins \\ Maria Camilla Bergonzi \\ Department of Chemistry
}

Vieri Piazzini, Elisa Landucci, Mario D'Ambrosio, laura tiozzo fasiolo, Lorenzo

Cinci, Gaia Colombo, Domenico Edoardo Pellegrini-Giampietro, anna rita bilia, Cristina Luceri, Maria Camilla Bergonzi

Carmelo Puglia, Silvia Fossati, Ylenia Zambito

\section{Submission Files Included in this PDF}

\section{File Name [File Type]}

Cover letter R1 IJBM.docx [Cover Letter]

Replies to Editor and Reviewers.docx [Response to Reviewers]

AbstractR1.docx [Abstract]

IJBIOMAC_2018_6721R1.docx [Manuscript File]

Captions to figures.docx [Figure]

FIG 1 REVISED.tiff [Figure]

FIG 2 REVISED_2.tiff [Figure]

Fig. 6.tif [Figure]

FIG 7_REVISED.pptx [Figure]

FIG 10.tiff [Figure]

Figure 13.tif [Figure]

Figure 14.tif [Figure] 


\section{Submission Files Not Included in this PDF}

File Name [File Type]

FIG 3_REVISED.xls [Figure]

FIG 4_REVISED.xIs [Figure]

FIG 5_REVISED.xls [Figure]

FIG 8_REVISED.xls [Figure]

FIG 9_REVISED.xls [Figure]

FIG 11_REVISED.xls [Figure]

Fig.12_REVISED.xIsx [Figure]

FIG 15_REVISED.xls [Figure]

To view all the submission files, including those not included in the PDF, click on the manuscript title on your EVISE Homepage, then click 'Download zip file'. 


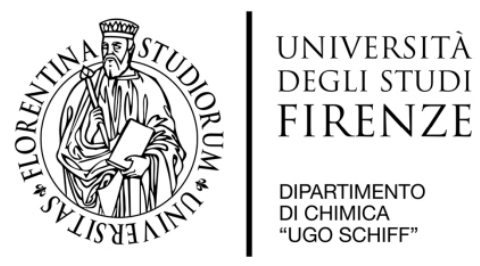

Dear Editor,

Please find attached the revised manuscript entitled "Chitosan coated human serum albumin nanoparticles: a promising strategy for nose-to-brain drug delivery" (IJBIOMAC_2018_6721), that my colleagues and me would submit for consideration for the publication in International Journal of Biological Macromolecules.

The manuscript was entirely revised according to reviewers' and Editor's comments. The revisions have been highlighted in yellow in the manuscript. In addition, the manuscript was corrected by a native English speaker and the corrections have been written in red.

All authors have read and approved this version of the article, and no part of this paper has been published nor is it submitted for publication elsewhere.

We thank you in advance for consideration.

Best regards.

Prof. Maria Camilla Bergonzi, Ph.D.

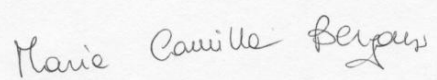




\section{Comments from the editors and reviewers:}

\section{-Editor}

As IJBIOMAC is a journal focusing on biological macromolecules, authors are required to provide basic characteristics of biomacromolecules used in the study, rather than simply stating they were purchased from suppliers or simply citing authors' previous paper, without detailed information about characteristics. The basic characteristics include molecular weight, viscosity, substitution degree (if applicable), structure and composition (if applicable), deacetylation degree (for chitosan), etc.

The characteristics of human serum albumin, mucin and chitosan were reported.

\section{-Reviewer 1}

The research paper describes the Chitosan coated human serum albumin nanoparticles: a promising strategy for nose-to-brain drug delivery. This work is methodically carried out and well written. In my opinion, the paper needs further improvements before being considered for publication according to the following comments/suggestions.

1. There are several papers on chitosan coated nanoparticles for the nose to brain delivery. Please state novelty of this work.

The novelty of our study was highlighted in the introduction.

2. Chemical crosslinker (glutaraldehyde) used is toxic in nature. Is there any other crosslinker can be used for safety?

The reviewer is right; there are several compounds able to perform the cross-linking reaction, besides glutaraldehyde: the enzyme transglutaminase, the carbodiimide and the natural compound genipin. In a previous paper (ChemMedChem 2016, 11, 1 - 11) the authors also tested a lesser-known thermal cross-linking method to prepare albumin nanoparticles.

However, glutaraldehyde as cross-linker is widely used to stabilize the albumin nanoparticles by crosslinking of amino acids residues in the protein (Int. J. Biol. Macromol. 115 (2018) 83-89).

Glutaraldehyde-crosslinked albumin behaves as a network to hold the drug molecules inside the nanoparticles (Colloids Surf. B: Biointerfaces 123 (2014) 286-292).

Glutaraldehyde forms a very stable aldehyde bond which prevents burst release of drug from the nanoparticle after the administration (K.A. Gawde et al. Colloids and Surfaces B: Biointerfaces 167 (2018) 8-19. Moreover, the Glutaraldehyde generates intra- and inter-molecular covalent bridges that increased the stability of NPs.

In the procedure reported by the authors, glutaraldehyde was used in the lowest possible concentration, useful to harden the coacervate and corresponding to $100 \%$ of the amount required for the cross-linking of the 60 amino groups of HSA. Among the different tested concentrations (from $50 \%$ to $200 \%$ of the calculated amount required for the cross-linking of the amino groups of HSA), the best results concerning particle size, homogeneity, yield and reproducibility were obtained with the $100 \%$ concentration 
Besides, the in vitro studies with two different cell lines, Caco-2 and $h C M E C / D 3$, have supported the biocompatibility of the formulation.

3. Authors should add rpm in below mention sentences

"Afterwards, $5 \mathrm{~mL}$ of CS solution was added to an equal volume of HSA NPs suspension under magnetic stirring at a speed of 250 at room temperature.

rpm was added.

4. Procedure for TEM studies needs to be included.

The procedure was added

5. Table 2 legends should come at top of the table.

The legends of the Tables have been moved at the top of the Tables.

6. Authors should include release mechanism of a model drug from nanocarriers such as fickian or non fickian or case transport mechanism after applying korsenmayer peppas model.

The $\mathrm{n}$ values were reported.

7. Authors evaluated "The surface morphology on NPs by using transmission electron microscopy"; but the authors can do dimensional analysis as well with TEM and can correlate with PCS. If there is a discrepancy between mean particle size determined by PCS and the particles shown in the TEM, authors can discuss these findings as shown in various research papers. Tzeyung et al., Pharmaceutics. 2019 Jan 10;11(1).

As reported in the "Results and discussion" (pag 14) TEM analysis of HAS NPs confirms the presence of spherical HSA NPs with uniform size distribution in the range of 230-250 nm, according to the PCS measurements. In the case of CS-HAS NPs, the same correlation between TEM and PCS data was found and made explicit in the text (pag 15). The small difference in the data obtained with the two techniques is discussed according to literature.

8. Authors can remove figure 2 as there was no any comparison between drug-loaded nanoparticle and drug suspension for in vitro release study. Authors can include the value in the text.

I did not understand what the reviewer means. The figure 2 (TEM image of CS-HAS NPs) is now modified according to reviewer's 2 comments and it was reported in the manuscript to highlight the presence of CS coating compared to uncoated particles and to confirm the DLS data. As reported in tables 1 and 2, the presence of the fluorescent model drug SalB does not affect the sizes of the carrier. In the in vitro release study (Fig 5) release profiles of SulfB solution and SulfB-loaded NPs were showed. The probe is soluble in water.

\section{There are many places typo, grammatical and syntax error need to be corrected.}

The manuscript was entirely revised. 


\section{-Reviewer 2}

- 1. In TEM image, please add highly populated nanoparticles image with proper magnification.

TEM images (Figure 1 and 2) were modified.

2. All figures are not appearing in the manuscript in pdf file.

As reported in the Guide for authors, in the "Formats" section, if the electronic artwork is created in a Microsoft Office application (Word, PowerPoint, Excel) the images can be supplied in the native document format. Some figures of our manuscript are loaded in the .xls or .ppt format. These figures are not present in the pdf file, but as separated files. 


\begin{abstract}
The aim of the present study was the development of human serum albumin nanoparticles (HSA NPs) as nose-to-brain carrier. To strengthen, the efficacy of nanoparticles as drug delivery system, the influence of chitosan (CS) coating on the performance of HSA NPs was investigated for nasal application. HSA NPs were prepared by desolvation technique. CS coating was obtained adding the CS solution to HSA NPs. The mean particle size was $241 \pm 18 \mathrm{~nm}$ and $261 \pm 8 \mathrm{~nm}$ and the $\zeta$-potential was $-47 \pm 3 \mathrm{mV}$ and $+45 \pm 1 \mathrm{mV}$ for HSA NPs and CS-HSA NPs, respectively. The optimized formulations showed excellent stability upon storage both as suspension and as freeze-dried product after 3 months. The mucoadhesion properties were assessed by turbidimetric and indirect method. NPs were loaded with sulforhodamine B sodium salt as model drug and the effect of CS coating was investigated performing release studies, permeation and uptake experiments using Caco-2 and hCMEC/D3 cells as model of the nasal epithelium and blood-brain barrier, respectively. Furthermore, ex vivo diffusion experiments have been carried out using rabbit nasal mucosa. Finally, the ability of the formulations to reversibly open tight and gap junctions was explored by western blotting and RTPCR analyzing in both Caco-2 and hCMEC/D3 cells.
\end{abstract}




\section{Chitosan coated human serum albumin nanoparticles: \\ a promising strategy for nose-to-brain drug delivery.}

Vieri Piazzini ${ }^{1 \neq}$, Elisa Landucci2 ${ }^{2 \neq}$, Mario D’Ambrosio ${ }^{3}$, Laura Tiozzo Fasiolo ${ }^{4,5}$, Lorenzo Cinci $^{3}$, Gaia Colombo ${ }^{5}$, Domenico E. Pellegrini-Giampietro ${ }^{2}$, Anna Rita Bilia ${ }^{1}$, Cristina Luceri $^{3}$, Maria Camilla Bergonzi ${ }^{1 *}$

${ }^{1}$ Department of Chemistry, via U. Schiff 6, 50519 Sesto Fiorentino, Florence, Italy ${ }^{2}$ Department of Health Sciences, Section of Clinical Pharmacology and Oncology, Viale Pieraccini 6, 50139 Florence, Italy.

${ }^{3}$ Department of NEUROFARBA, Department of Neurosciences, Psychology, Drug Research and Child Health, Section of pharmacology and Toxicology, Viale Pieraccini 6, 50139 Florence, Italy.

${ }^{4}$ Department of Department of Food and Drug, via delle Scienze 27/A, 43124 Parma, Italy.

${ }^{5}$ Department of Department of Life Sciences and Biotechnology, Via Fossato di Mortara 17/19, 44121 Ferrara, Italy

$\neq$ These authors contributed equally to this work.

\section{Corresponding author}

Prof. Maria Camilla Bergonzi, University of Florence, Department of Chemistry, via U. Schiff 6, 50019 Sesto Fiorentino, (FI), Italy.

E-mail: mc.bergonzi@unifi.it phone: +39 055-457 3678. 


\begin{abstract}
The aim of the present study was the development of human serum albumin nanoparticles (HSA NPs) as nose-to-brain carrier. To strengthen, the efficacy of nanoparticles as drug delivery system, the influence of chitosan (CS) coating on the performance of HSA NPs was investigated for nasal application. HSA NPs were prepared by desolvation technique. CS coating was obtained adding the CS solution to HSA NPs. The mean particle sizes was $241 \pm 18 \mathrm{~nm}$ and $261 \pm 8 \mathrm{~nm}$ and the $\zeta$-potential was $-47 \pm 3 \mathrm{mV}$ and $+45 \pm 1 \mathrm{mV}$ for HSA NPs and CS-HSA NPs, respectively. The optimized formulations showed excellent stability upon storage both as suspension and as freeze-dried product after 3 months. The mucoadhesion properties were assessed by turbidimetric and indirect method. NPs were loaded with sulforhodamine B sodium salt as model drug and the effect of CS coating was investigated performing release studies, permeation and uptake experiments using Caco-2 and hCMEC/D3 cells as model of the nasal epithelium and blood-brain barrier, respectively. Furthermore, ex vivo diffusion experiments have been carried out using rabbit nasal mucosa. Finally, the ability of the formulations to reversibly open tight and gap junctions was explored by western blotting and RT-PCR analyzing in both Caco-2 and hCMEC/D3 cells.
\end{abstract}

\title{
Keywords
}

Albumin nanoparticles; Chitosan; Caco-2 cell; hCMEC/D3 cells; rabbit nasal mucosa; nose to brain. 


\section{Introduction}

Most of the drugs, in particular hydrophilic molecules and high molecular weight compounds, are not favourably absorbed by the brain due to the presence of the blood-brain barrier (BBB) and blood-cerebrospinal fluid barrier, that cause a selective permeability to circulating molecules $[1,2]$. In the last years, the nose-to-brain (NTB) delivery is emerging strategy to overcome these barriers and delivery the drug directly into the brain. An increasing number of products are being developed and are reaching the market, such as vaccines, painkillers, antimigraine and anti-cancer drugs and hormones $[3,4]$.

The intranasal route allows the administration of drugs both locally and systemically avoiding the typical gastrointestinal degradation of oral administration and the effect of hepatic metabolism. The rich vascularization of the nasal mucosa provides a series of unique attributes that can increase the safety, the patient compliance, the rate of absorption of the drug and, consequently, the speed of onset of the therapeutic effect. Furthermore, the nasal mucosa, is easily accessible compared to other membranes and it is a favourable entry route for both small and large molecules. The nasal bioavailability of small molecules is good and the drugs that are not absorbed orally can be conveyed to the nasal systemic circulation. Nasally administered drugs can rapidly reach the brain through different pathways: the first is the olfactory nerves, that represent the main direct pathway for the NTB drug delivery; then the trigeminal nerves, which have nerve endings in the respiratory epithelia; finally, the respiratory epithelium through which the drugs reach the circulation and subsequent cross the BBB $[1,5,6]$.

However, the NTB delivery is characterized by several limitations such as the small volume of the nasal cavity, the mucociliary clearance, the enzymatic degradation, the low drug retention time, the potential nasomucosal toxicity, the technique of drug administration and deposition and the necessity of a suitable delivery device $[4,7,8]$.

To overcome these drawbacks, pharmaceutical nanotechnologies appear as an ideal formulation strategy for the NTB delivery [1,4,6-9]. In this context, the aim of the present study was the development of human serum albumin nanoparticles (HSA NPs) as NTB carrier and the investigation of the effect of chitosan (CS) coating on their performance, in particular with respect to the surface and mucoadhesive properties, stability, drug release modulation, biocompatibility and permeability.

HSA is an endogenous protein, non-toxic, non-immunogenic, biodegradable and biocompatible. Moreover, it displays good mucoadhesive properties, suitable for prolonging the residence time in the nasal cavity. HSA enhances cellular uptake by its active targeting 
properties and it can show conformational modifications to increase its stability $[11,12]$. Furthermore, as reported in literature, albumin is an ideal substance for the development of nanocarriers with the aim of increasing bioavailability, stability and pharmacological effects of synthetics and plant-derived molecules [13-18].

HSA NPs were employed for the NTB delivery of the anti-Alzheimer drugs tacrine and $R$ flurbiprofen $[19,20]$.

CS, a polysaccharide derived from deacetylation of chitin, was also employed for its ability to reversibly open tight junctions, with the potential to increase the drug permeation across the nasal mucosa and the extracellular transport along the olfactory and trigeminal nerves. Besides, positively charged CS can increase the drug residence time of the loaded drug in the nasal cavity, due to the electrostatic interactions with the negatively charged epithelial cells or the ability to absorb water from the mucus layer, forming a gel-like layer that increases the contact with the site of absorption $[4,21,22,23]$. CS NPs and other CS derived formulations were investigated for nose to brain delivery [22]

Various HSA and CS NPs have been utilized to deliver anticancer agents at the tumor. Proteins and polysaccharides-based nanocarriers have great potential as drug delivery based on their biocompatibility, biodegradability, ease of functionalization, improved biodistribution and reduced toxicity effects $[21,24,25]$. The main application of CS coated formulations is for the oral administration. CS-coated PLGA and PCL NPs were considered for nasal delivery [5,26]. CS-coated HSA (CS-HSA NPs) nanoparticles functionalized by MUC1 aptamer have previously reported to load paclitaxel and investigate the specific targeting potential in human breast tumor cells, MCF7 and T47D [21]. However, the present study is the first application of CS-HSA NPs for NTB delivery. The surface charges modification of NPs from negative, with HAS, to positive in the presence of CS have also considered due to its important effect on the interaction with cell membranes and on cellular uptake [21].

Developed HAS NPs and CS-HAS NPs were physically and chemically characterized by Light Scattering techniques (DLS and ELS), Transmission Electron Microscopy and High Performance Liquid Chromatography. The freeze-drying process was evaluated as technique to increase NPs stability. The mucoadhesion properties were assessed through the turbidimetric method and the evaluation of the change in the $\zeta$-potential value. HSA NPs and CS-HSA NPs were loaded with sulforhodamine B sodium salt (SulfB) as a model of a hydrophilic drug (SulfB-HAS NPs and SulfB-CS-HAS NPs) and the effect of CS coating was investigated with respect to the release behaviour, the cellular permeation and the uptake using Caco-2 and 
hCMEC/D3 cells as model of the nasal epithelium and BBB, respectively [15,23,27-30]. Furthermore, ex vivo diffusion experiments were performed using rabbit nasal mucosa in a Franz-type permeation apparatus. The possible effects of the two formulations on tight and gap junctions expression were assessed by exposing Caco-2 and hCMEC/D3 cells to HSA NPs and CS-HSA NPs and by measuring the expression of Tight Junction Protein 1 (TJP1, also known as zona occludens protein-1), at the protein and RNA levels.

\section{Material and methods}

\subsection{Material}

Sigma-Aldrich (Milan, Italy) provided all chemicals, analytical grade and HPLC grade solvents. Human serum albumin (Sigma-Aldrich, Milan, Italy; cat n. SPR6182, mol wt 66.4 kDa). Chitosan low molecular weight (Sigma-Aldrich, Milan, Italy; cat n. 448869, mol wt. 50,000-190,000 Da, viscosity $20-300 \mathrm{cP}, 1$ wt. \% in $1 \%$ acetic acid, $25{ }^{\circ} \mathrm{C}$, Brookfield). Mucin from porcine stomach (Sigma-Aldrich, Milan, Italy; cat n. M1778, Type III, bound sialic acid

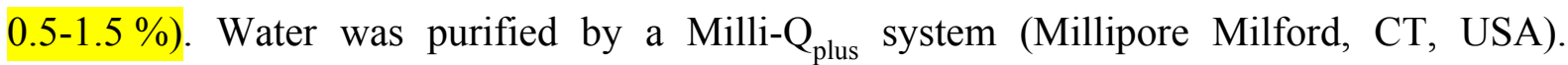
Phosphotungstic acid (PTA) was from Electron Microscopy Sciences (Hatfield, PA, USA). Dialysis kit was from Spectrum Laboratories, Inc. (Breda, The Netherlands).

\subsection{Methods}

\subsubsection{Preparation of human serum albumin nanoparticles}

HSA NPs were prepared by the desolvation-coacervation technique [31,32], with some modifications. HSA (30 mg) was dissolved in $3 \mathrm{~mL}$ of $10 \mathrm{mM} \mathrm{NaCl}$ solution under magnetic stirring. The $\mathrm{pH}$ value was adjusted between 8 and 9 with $\mathrm{NaOH} 0.1 \mathrm{~N}$. Then, ethanol was added at a constant rate using a syringe until turbidity appeared in the solution. The obtained coacervates were hardened by the addition of $5 \%$ glutaraldehyde solution, corresponding to $100 \%$ of the amount required for the cross-linking of the 60 amino groups of HSA. After $2 \mathrm{~h}$ under magnetic stirring at room temperature, ethanol was vacuum evaporated, and the pellet was redispersed in distilled water.

In addition, fluorescent NPs (SulfB-HAS NPs) were prepared, adding sulforhodamine B sodium salt $\left(\lambda_{\mathrm{ex}}=565 \mathrm{~nm}, \lambda_{\mathrm{em}}=586 \mathrm{~nm}\right.$ in water, SulfB) to the HSA solution (final concentration $0.1 \mathrm{mg} / \mathrm{mL}$ ). The resulted mixture was incubated for $2 \mathrm{~h}$ under magnetic stirring at $500 \mathrm{rpm}$, before the addition of ethanol [11]. 


\subsubsection{Preparation of chitosan-coated nanoparticles}

CS-HSA NPs were obtained exploiting the ionic interactions between negatively charged HSA NPs and positively charged CS [21]. Firstly, CS was dissolved in 1\% v/v glacial acetic acid at the concentration of $1 \mathrm{mg} / \mathrm{mL}$. After $24 \mathrm{~h}$ under stirring at room temperature, the solution was filtered through a $0.45 \mu \mathrm{m}$ membrane and then, the $\mathrm{pH}$ was adjusted to 4.5 with $\mathrm{NaOH}$ diluted solution $(0.1 \mathrm{~N})$. Afterwards, $5 \mathrm{~mL}$ of CS solution was added to an equal volume of HSA NPs suspension under magnetic stirring at a speed of $250 \mathrm{rpm}$ at room temperature. The stirring was maintained for $15 \mathrm{~min}$ to allow the stabilization of the CS coating.

SulfB-CS-HSA NPs were prepared by adding CS solution to SulfB-HAS NPs suspension.

\subsubsection{Physical characterization}

Particle size, the polydispersity index (PdI) and $\zeta$-potential have been measured a Zsizer Nanoseries ZS90 instrument (Malvern Instrument, Worcestershire, UK). The analyses were performed in triplicate.

\subsubsection{TEM}

The morphological characterization of NPs was analyzed by transmission electron microscopy (Jeol 1010, Tokyo, Japan). The samples were prepared as previously reported [29]. They were properly diluted with distilled water, then they were dropped on a 200 mesh carbon filmcovered copper grid and negatively stained with a phosphotungstic acid aqueous solution (1\% $\mathrm{w} / \mathrm{v})$. After drying, the grid containing the samples were observed with the TEM.

\subsubsection{NPs yield determination}

In order to evaluate the amount of HSA transformed into nanoparticles, $1.5 \mathrm{~mL}$ of HSA NPs and CS-HSA NPs were centrifuged at $21773 \mathrm{x}$ g for $45 \mathrm{~min}$. Then, the obtained supernatants were analyzed by HPLC [33].

The chromatograph was a HP 1200 liquid chromatograph coupled with a diode-array-detector (DAD) (Agilent Technologies, Santa Clara, CA, USA). The column was a Luna Omega Polar $(150 \mathrm{~mm} \times 3 \mathrm{~mm}, 5 \mu \mathrm{m})$ (Agilent Technologies, Santa Clara, CA, USA). The mobile phases were: (A) formic acid/water $\mathrm{pH} 3.2$ and (B) acetonitrile. The flow rate was $0.5 \mathrm{~mL} / \mathrm{min}$. The multi-step linear solvent gradient applied was: 0-2 min, 90\% A, 10\% B; 2-7 min, 90-60\% A, $10-40 \%$ B; 7-17 min, 60-30\% A, 40-70\% B; 17-20 min, 30-10\% A, 70-90\% B; 20-22 min 10\% A, $90 \%$ B; $22-27$ min, 10-90\% A, 90-10\% B with post-time of 8 min. UV detection was carried 


\subsubsection{Nanoparticle freeze-drying}

An aliquot of HSA NPs and CS-HSA NPs was lyophilized as such, other three aliquots were freeze-dried in the presence of D-Glucose anhydrous or D-Mannitol or D-Sucrose as cryoprotective agents $(2 \% \mathrm{w} / \mathrm{v})$ [34]. After the freeze-drying process, each sample was reconstituted with the original volume of distilled water, vortexed for few minutes to ensure the complete redispersion of the powder [34] and analyzed for particle size, polydispersity and $\zeta$ potential.

\subsubsection{Stability studies}

Stability studies were conducted for three months. Particle size, PdI and $\zeta$-potential were evaluated at $4^{\circ} \mathrm{C}$ for HSA NPs and CS-HSA NPs suspensions, and at room temperature for freeze-dried products.

\subsubsection{Mucoadhesion properties}

To evaluate and compare the mucoadhesion properties of both NPs in the presence of mucin, the turbidimetric method [35] and the evaluation of the $\zeta$-potential (indirect method) were applied [36,37]. An equal volume of nanoparticles suspension and 1\% w/v mucin aqueous solution were mixed. Then, the resulted samples were vortexed for $1 \mathrm{~min}$ and incubated in a 
water bath for $1 \mathrm{~h}$ at $37^{\circ} \mathrm{C}$. The turbidity of the mixtures was evaluated spectrophotometrically at $\lambda=500 \mathrm{~nm}$. The absorbances of individual mucin and nanoparticle suspensions were also recorded. The interaction between mucin and HSA NPs or CS-HSA NPs was calculated using the following equation:

$$
\Delta \mathrm{A}=\mathrm{A}-\mathrm{A}_{\text {theor }}
$$

where $\Delta \mathrm{A}$ is the absorbance difference, $\mathrm{A}$ is the effective absorbance of HSA NPs-mucin and CS-HSA NPs-mucin mixtures and $\mathrm{A}_{\text {theor }}$ represents the sum between the individual absorbance of mucin and nanoparticles suspensions. If $\Delta \mathrm{A}=0$, no interactions occur, whereas if $\Delta \mathrm{A}>>0$ there is a strong interaction between nanoparticles and mucin.

The $\zeta$-potential was measured as previously reported.

\subsubsection{In vitro release studies}

Dialysis bag method was applied for the in vitro release studies in physiological $\mathrm{pH}$ conditions (PBS pH 7.4). For these studies regenerated cellulose membranes (cut-off of $3.5 \mathrm{kD}$ ) were chosen for retaining the nanoparticles and allowing for the diffusion of the entrapped compound into the dissolution medium. SulfB-HAS NPs, SulfB-CS-HSA NPs or SulfB aqueous solution $(2 \mathrm{~mL})$ were added into dialysis membranes and placed in $200 \mathrm{~mL}$ of release medium. Thus, the systems were incubated at $37^{\circ} \mathrm{C}$ with stirring at $150 \mathrm{rpm}$. At predetermined time intervals $(0,0.5,1,2,4,5,8$ and $24 \mathrm{~h}) 1 \mathrm{~mL}$ of the medium was withdrawn and replaced with the same volume of fresh release medium maintained at $37^{\circ} \mathrm{C}$ to preserve sink conditions. SulfB released at each time interval was quantified using HPLC. All experiments were performed in triplicate. Different mathematical models were used to understand the kinetic and the mechanism of drug release from HSA NPs and CS-HSA NPs. The best-fitted model was defined based on the highest $\mathrm{R}^{2}$ value of the release curve.

\subsubsection{Experiments with Caco-2 cells}

Caco-2 cell line

Caco-2 cells (human colorectal adenocarcinoma) were from American Tissue Type Culture Collection (Manassas, VA, USA) and cultured in Dulbecco's modified Eagle's medium with $20 \%$ fetal bovine serum (FBS) and $100 \mathrm{U} / \mathrm{mL}$ penicillin-streptomycin in $5 \% \mathrm{CO}_{2}$ at $37{ }^{\circ} \mathrm{C}$ (Thermo Fisher Scientific, Rodano, Milan, Italy). 


\section{Cell Culture Experiments}

The cell viability was assessed using MTS assay, as previously described [38]. The cells were exposed to HSA NPs and CS-HSA NPs at different dilutions (from 1:10 to 1:40) for 2 and 24 $\mathrm{h}$. The relative cell viability was expressed as a percentage of the untreated control group.

\section{Transport Studies}

Caco-2 cells were seeded into 12-well PET transwell plates $\left(1.13 \mathrm{~cm}^{2}\right.$ growth surface area and pore size $0.4 \mu \mathrm{m}$, Greiner Bio-One, Milan, Italy) at a density of $2 \times 10^{4}$ cells $/ \mathrm{cm}^{2}$. A confluent monolayer was obtained after 21 days.

SulfB-HSA NPs and SulfB-CS-HSA NPs diluted 10 times were incubated for $4 \mathrm{~h}$ in the apical compartment. Lucifer Yellow (LY) was used to check the integrity of the cellular layer during the permeability test [39]. At 0 (before sample addition), 60, 120, 180 and $240 \mathrm{~min}$, samples were taken from the basal compartment of each transwell plate and fresh HBSS was added. The SulfB concentration was determined by HPLC.

\section{Mucin staining}

To highlight the presence of mucin production on the Caco-2 cells monolayer, cells were seeded and cultured for 21 days in specific chamber-slide for cytological analysis ( $\mathrm{X}$-well tissue culture chambers, Sarstedt, Verona, Italy). After 21 days of culture, cells were fixed in 4\% paraformaldehyde for 15 minutes at room temperature. Slides were rinsed in 3\% acetic acid solution and incubated for 2 hours in $1 \%$ alcian-blue solution, then, were rinsed in $\mathrm{H}_{2} \mathrm{O}$, oxidized for 10 minutes in $1 \%$ periodic acid solution and incubated for 10 minutes in Schiff reactive. Slides were finally rinsed in $\mathrm{H}_{2} \mathrm{O}$ and washed three times in $0.5 \%$ sodium bisulfite solution, dehydrated and mounted. Nuclei were counterstained with hematoxylin for 30 seconds.

\section{Cell uptake Studies}

Caco-2 cells were pre-incubated with sodium azide $(1 \mu \mathrm{M})$, chlorpromazine $(15 \mu \mathrm{M})$, and indomethacin $(25 \mu \mathrm{M})$ for 30 min followed by the addition of SulfB-HSA NPs or SulfB-CSHSA NPs 1:10 for $1 \mathrm{~h}$, or maintained at $4^{\circ} \mathrm{C}$ during the NPs exposure. At the end of the experiments, the SulfB quantity remaining in the cellular lysate was quantified by HPLC analyses. 


\subsubsection{Experiments with hCMEC/D3 cells}

hCMEC/D3 cell line

The brain microvascular endothelial cell line hCMEC/D3 (Millipore) derives from human temporal lobe microvessels isolated from epilepsy control obtained by medical surgery. Cell were seeded and grown as previously reported [29]. Cell were maintained at $37^{\circ} \mathrm{C}$ in an incubator in atmosphere of humidified air and $5 \% \mathrm{CO}_{2}$ and the EndoGRO medium supplemented with $1 \mathrm{ng} / \mathrm{mL}$ FGF-2 was changed thrice a week. Cells were passaged at least twice before use. Accumax TM Cell Counting Solution in DPBS was used to split the confluent cells.

\section{MTT assay}

The cell viability after HSA NPs and CS-HSA NPs exposure in hCMEC/D3 cell was assessed by MTT assay [40,41]. After pre-coated of the 24-well plate with Collagen Type I, Rat Tail, the cells were seeded and maintained until they were approximately $70-80 \%$ confluent. HSA NPs and CS-HSA NPs were incubated at different concentrations (diluition 1:10, 1:100 and 1:500) of the formulation for 2 and $24 \mathrm{~h}$ in the complete medium (EBM-2). A part of the medium of each treatment was taken and preserved for LDH assay, and cells were incubated with MTT at the concentration of $1 \mathrm{mg} / \mathrm{mL}$. Finally, DMSO was used to dissolve MTT formation and absorbance was recorded at 550 and $690 \mathrm{~nm}$. EBM-2 was used as posite control and Triton $\mathrm{X}-100$ as negate control and the cell viability was expressed as a percentage compared to the cells incubated only with EBM-2.

\section{LDH assay}

The extent of cell death after HSA NPs and CS-HSA NPs exposure was quantitatively evaluated by measuring the amount of LDH release from injured cells, as previously described [29]. The LDH level corresponding to complete cell death was obtained in the presence of triton X-100 (positive control). All the values were expressed as a percentage compared to TX release, EBM2 was used as the negative control.

\section{hCMEC/D3 cell culture for transwell permeability studies}

High-density pore $\left(2 \times 10^{6}\right.$ pores $\left./ \mathrm{cm}^{2}\right)$ transparent PET membrane filter inserts $(0.4 \mu \mathrm{m}, 23,1$ mm diameter, Falcon, Corning BV, Netherlands) in 6-well plates were used for all transcytosis assays as previous described [29]. After $1 \mathrm{~h}$ of coating with rat tail collagen type I the membrane 
filter inserts, the cells were seeded onto the apical side of the inserts at a density of $6 \times 10^{4}$ cells $/ \mathrm{cm}^{2}$. hCMEC/D3 monolayers were used to perform the permeability assay for SulfB-HSA NPs and SulfB-CS-HSA NPs. The integrity of the monolayer cells was controlled using fluorescein sodium salt $(\mathrm{NaF})$ at a concentration of $10 \mu \mathrm{g} / \mathrm{mL}$ [15], and by observation of cultures under phase-contrast microscopy [28] using an inverted microscope (Olympus IX-50; Solent Scientific, Segensworth, UK) with (20X). For permeability studies, SulfB-HSA NPs and SulfB-CS-HSA NPs, diluted 10 times were tested and incubated for 1, 2 and $3 \mathrm{~h}$ in the apical donor compartment. NaF and SulfB were quantified in both in compartments by HPLC-FLD or HPLC-DAD analyses.

\section{Cell uptake Studies}

Sodium azide $(1 \mu \mathrm{M})$, chlorpromazine $(15 \mu \mathrm{M})$ and indomethacin $(25 \mu \mathrm{M})$ were used to understand the uptake mechanism of the developed nanoparticles. The drugs were preincubated for $30 \mathrm{~min}$ before the addition of SulfB-HSA NPs or SulfB-CS-HSA NPs for $2 \mathrm{~h}$ at the dilution of 1:10. The quantity of SulfB was measured on cellular lysate by HPLC analyses at the end of the test.

\section{Western blotting}

hCMEC/D3 and Caco-2 cells were seeded into 6-well plates at a density of $1.2 \times 10^{6}$ cells $/ \mathrm{cm}^{2}$ and after exposure to HSA NPs and CS-HSA NPs at the dilution of 1:10 for $2 \mathrm{~h}$, the cells (1 well/sample) were washed with cold 0.01 M PBS, pH 7.4 and were transferred and dissolved in a tube containing 1\% SDS. The Pierce (Rockford, IL, USA) BCA (bicinchoninic acid) Protein Assay Total was used to quantify the protein levels. Lysates (20 $\mu \mathrm{g} / \mathrm{lane}$ of protein) were resolved by electrophoresis on a 4-20\% SDS-polyacrylamide gel (Bio-Rad Laboratories, Hercules, CA, USA) and transferred onto nitrocellulose membranes. Blots were blocked for 1 $\mathrm{h}$ at room temperature in $20 \mathrm{mM}$ Tris-buffered saline, $\mathrm{pH} 7.6-0.1 \%$ Tween 20 (TBS-T) containing $5 \%$ non-fat dry milk, and then incubated overnight at $4{ }^{\circ} \mathrm{C}$ with rat monoclonal antibodies against ZO1, mouse monoclonal antibodies against claudin-5 (all from Santa Cruz Biotechnology, CA, U.S.A) diluted 1:300 in TBS-T containing 5\% non-fat dry milk or polyclonal-rabbit antibody against Ve-Chaderin (from Cell Signaling Technology, Beverly, MA, USA) diluted 1:1000 in TBS-T containing 5\% bovine serum albumin. The loading control anti- $\beta$-actin antibody was monoclonal from Sigma (St Louis, MO, USA). Immunodetection was performed with secondary antibodies (1:2000 anti-rat, anti-mouse or anti-rabbit IgG from 
donkey, Amersham Biosciences, UK) conjugated to horseradish peroxidase in TBS-T containing 5\% non-fat dry milk. Membranes were washed with TBS-T and then reactive bands were detected using chemiluminescence (ECLplus; Euroclone, Padova, Italy). Quantitative analysis was performed using the QuantityOne analysis software (Bio-Rad, Hercules, CA, USA).

\section{$R T-P C R$}

hCMEC/D3 and Caco-2 cells were seeded into 12-well plates at a density of $1.0 \times 10^{5} \mathrm{cells} / \mathrm{cm}^{2}$. After exposure to HSA NPs and CS-HSA NPs at the dilution of 1:10 for $2 \mathrm{~h}$, the cells were washed with Dulbecco-s phosphate-buffered saline. The Nucleo Spin ${ }^{\circledR}$ RNA kit (MachereyNagel, Bethlehem, USA) were used to extractthe total RNA from cell lysates.

For first-strand cDNA synthesis, $1 \mu \mathrm{g}$ of total RNA from each sample was reverse-transcribed by using the RevertAid RT Kit (Thermo Scientific, Waltham, MA USA). Primers were designed on the basis of the GenBank sequences for homo sapiens Tight Junction Protein 1, TJP1 (NM_003257.4): forward primer 5'-GGGAGCACATGGTGAAGGTAA-3', reverse primer 5'- ATCACAGTGTGGTAAGCGCA-3'. GAPDH was co-amplified as reference gene: forward primer 5'-CCCTCAAGGGCATCCTGGGCT-3', reverse primer 5'GCAGGGACTCCCCAGCAGTGA-3'. PCRs were carried out using $1 \mu$ of cDNA in a $25 \mu 1$ total volume containing $1 \mathrm{x}$ PCR buffer, $0.5 \mathrm{mM}$ dNTPs, $8 \mathrm{ng} / \mu \mathrm{l}$ of primer, $0.1 \mathrm{ng} / \mu \mathrm{l}$ of GAPDH primers and 1.25 units of Taq polymerase (Dream Taq, Carlo Erba, Milan, Italy). The PCR conditions were: $95^{\circ} \mathrm{C}$ for $5 \mathrm{~min}$ and 30 cycles at $95^{\circ} \mathrm{C}$ for $30 \mathrm{sec}, 60^{\circ} \mathrm{C}$ for $30 \mathrm{sec}$ and $72^{\circ} \mathrm{C}$ for 55 sec. PCR products were separated on a agarose gel $(1.8 \%)$ and visualized by Safeview staining (Euroclone, Milan, Italy). Gel images were captured by an UVIdocHD2 acquired system (Eppendorf, Milan, Italy) and the intensity of the bands were analysed with the Quantity-One software (Bio-Rad, Segrate, Milan, Italy).

\subsubsection{Ex vivo transport experiments across rabbit nasal mucosa}

Ex vivo transport experiments were performed using rabbit nasal mucosa in a Franz-type diffusion apparatus $\left(0.58 \mathrm{~cm}^{2}\right.$ permeation area, VETROTECNICA S.r.1., Padova, Italy) [4244]. On the day of the experiment, rabbit heads were collected from a local slaughterhouse (Pola S.r.l, Finale Emilia, Italy) and transported to the laboratory in a refrigerated box. The extraction procedure, completed within $2 \mathrm{~h}$ from the animal's death, and the protocol to treat the nasal mucosa are described elsewhere [45]. 
Before introducing the NPs in the donor compartment, the receptor compartment was filled with PBS and the assembled system was allowed to equilibrate at $37^{\circ} \mathrm{C}$ for half an hour.

In particular, SulfB-HSA NPs, SulfB-CS-HSA NPs and SulfB aqueous solution were tested by maintaining the concentration of SulfB constant in all the samples $(0.1 \mathrm{mg} / \mathrm{mL})$.

The experiments were carried out over a $4 \mathrm{~h}$ and $24 \mathrm{~h}$ period of time. This experiment duration was consistent with the data in literature about tissue viability and drug transport experiments across animal excised nasal mucosa, employing nano-drug delivery systems [46-51].

At predetermined time points, a volume of receptor solution $(0.5 \mathrm{~mL})$ was withdrawn, and the receptor compartment refilled with an equivalent volume of fresh PBS. At the end of the experiment, the mass balance (sum of the amounts of SulB recovered from receptor, donor and membrane) was calculated. For this aim, the residual formulation on the membrane was quantitatively recovered by rinsing the donor compartment with PBS. In addition, SulfB retained at the membrane nasal mucosa was extracted with distilled water using an IKA T25 digital Ultra-Turrax (IKA-Works, Staufen im Breisgau, Germany) for $10 \mathrm{~min}$ and then an ultrasound bath for $30 \mathrm{~min}$. All the samples were analyzed by HPLC.

The flux of SulfB across the mucosa in steady-state conditions was calculated according to the solution of Fick Equation [43].

\subsubsection{Statistical analysis}

The experiments were performed in triplicate. The results were expressed as a mean \pm S.D. (standard deviation). Statistical analyses cells viability, permeability studies and cellular uptake were performed using one-way ANOVA followed by the post hoc Tukey's w-test for multiple comparisons. GRAPH-PAD PRISM v. 5 for Windows (GraphPad Software, San Diego, CA, USA) was applied to all statistical calculations. A P value $<0.05$ was considered significant.

\section{Results and Discussion}

\subsection{HSA NPs characteristics}

HSA NPs were obtained by the desolvation-coacervation technique [31,32]. The hydrodynamic diameter of the nanoparticles is one of the most crucial parameters that affect the nose-to-brain absorption. Indeed, only small particles can be transported to the brain via the olfactory or the trigeminal nerves [1]. For this reason, the $\mathrm{pH}$ of the HSA solution was adjusted between 8 and 
9 considering that the particle size decreased with increasing $\mathrm{pH}$ [32]. $\mathrm{NaCl}$ was selected because other salts interfere with the desolvation and cross-linking process [32,52].

Ethanol was used as desolvating agent due to its dielectric constant, dipole moment and solubility parameter that make it an ideal solvent to enter into the hydrophobic region of HSA, disrupt the hydrophilic layer of the protein in water, and to induce the denaturation of HSA and consequently the formation of NPs [53]. Besides, ethanol is less toxic compared to other organic solvents proposed as desolvating agents, i.e., acetone, methanol and acetonitrile [54,55].

Glutaraldehyde aqueous solution was chosen as cross-linker agent [32]. Among the different tested concentrations (from $50 \%$ to $200 \%$ of the calculated amount required for the crosslinking of the amino groups of HSA), the best results concerning particle size, homogeneity, yield and reproducibility were obtained with the $100 \%$ concentration (Table 1).

The morphological appearance of NPs confirmed the presence of spherical HSA NPs with uniform size distribution in the range of $230-250 \mathrm{~nm}$, according to the DLS measurements (Figure 1).

The surface charge of the developed HSA NPs was negative due to the presence of the terminal carboxyl groups of the protein. The high $\zeta$-potential value indicates the presence of strong repulsive forces which can prevent the aggregation of the NPs, increasing the stability of the formulation (Table 1). Besides, the negative surface charge is crucial for further surface modification step.

Table 1. Physical and chemical characterization of empty and SulfB-HSA NPs. Data displayed as mean $\pm \mathrm{SD} ; \mathrm{n}=3$.

\begin{tabular}{cccccc}
\hline Sample & Size $(\mathbf{n m})$ & PdI & ל-potential $(\mathbf{m V})$ & EE\% & Yield \% \\
\hline HSA NPs & $241 \pm 19$ & $0.06 \pm 0.04$ & $-47 \pm 3$ & - & $93 \pm 1$ \\
SulfB-HSA NPs & $244 \pm 12$ & $0.11 \pm 0.02$ & $-44 \pm 5$ & $65 \pm 2$ & $95 \pm 2$ \\
\hline
\end{tabular}

The nanoparticle yield, determined by HPLC analyses as previously reported in the literature [33], was very high, indicating that almost all HSA was converted into NPs. Thus, the obtained results demonstrated the success of the preparative process.

SulfB, a hydrophilic fluorescent dye, was incorporated into HSA NPs (SulfB-HSA NPs) as hydrophilic drug model to perform release studies and in vitro experiments with Caco-2 and hCMEC/D3 cells and to investigate the permeation across the rabbit nasal mucosa. SulfB was 
selected because it does not have a $\mathrm{pH}$-dependent absorption or fluorescence over the range of 3 to 10 and it is highly soluble in the media employed in the in vitro/ex vivo assays. The incorporation of SulfB did not negatively affect the size and homogeneity of HSA NPs, as reported in Table 1.

\subsection{CS-HSA NPs characteristics}

CS-HSA NPs were obtained by the electrostatic interaction between negatively charged HSA NPs and positively charged CS [21]. Low molecular weight CS was used according to Bonaccorso [5]. The increase in particle size and the inversion of $\zeta$-potential value clearly indicated the interaction between HSA NPs and CS [37] (Table 2). The high $\zeta$-potential is related to a stable formulation in which the electrostatic repulsions prevent the aggregation. The positive surface charge of CS-HSA NPs is also fundamental for further interaction with negatively charged cell membranes and to improve the mucoadhesive properties.

Different CS concentrations were tested (from $1 \mathrm{mg} / \mathrm{mL}$ to $3 \mathrm{mg} / \mathrm{mL}$ ). As CS concentration increased, particle enlargement (greater than $300 \mathrm{~nm}$ ) and broadening of the size distribution $(\mathrm{PdI}>0.3)$ were observed, while the $\zeta$-potential remained unchanged. Eventually, CS was employed at the concentration of $0.1 \mathrm{mg} / \mathrm{mL}$ (Table 2).

TEM micrographs provided information on morphology and dimensions of the NPs and showed the presence of CS on the surface of HSA NPs as a grey shell (Figure 2) [37,56,57]. Dimensional analysis with TEM (220-230 nm) correlated with DLS values. The little difference between the results obtained with the two analytical techniques could be due to different sample preparation: the dehydration of CS NPs in the case of TEM leads to measurement of the particle which is smaller than hydrodynamic particles, as previously reported [28]. The other physicochemical properties of HSA NPs such as PdI, EE\% and the nanoparticle yield were not affected by the presence of CS.

Table 2. Physical and chemical characteristics of empty and SulfB-CS-HSA NPs. Data displayed as mean $\pm \mathrm{SD} ; \mathrm{n}=3$.

\begin{tabular}{cccccc}
\hline Sample & Size (nm) & PdI & ל-Potential (mV) & EE\% & Yield \% \\
\hline CS-HSA NPs & $261 \pm 8$ & $0.10 \pm 0.07$ & $+45 \pm 1$ & - & $94 \pm 1$ \\
SulfB-CS-HSA NPs & $267 \pm 6$ & $0.12 \pm 0.02$ & $+44 \pm 1$ & $63 \pm 1$ & $95 \pm 3$ \\
\hline
\end{tabular}




\subsection{Nanoparticles freeze-drying}

The freeze-drying process improves the long-term stability of nanoparticles and limits the microbial proliferation caused by the presence of the aqueous medium. In this perspective, HSA NPs and CS-HSA NPs were freeze-dried without cryoprotectants and in the presence of sugars or sugar alcohols such as glucose, sucrose and mannitol. Fast freezing rate with liquid nitrogen was preferred because it avoids the formation of large ice crystals, which could damage the NPs and reduces their aggregation $[59,60]$. In the absence of cryoprotectant, a significant increase in size and a loss of size homogeneity were observed for both formulations (Table 3). This result confirmed the advantage of cryoprotectants in preventing the aggregation of albumin NPs during the freeze-drying process [34].

Table 3. Physical parameters of HSA NPs and CS-HSA NPs after the freeze-drying process without and with different cryoprotectants. Data displayed as mean $\pm \mathrm{SD} ; \mathrm{n}=3$.

\begin{tabular}{cccc}
\hline Freeze-dried product & Size (nm) & PdI & $\zeta$-potential $(\mathbf{m V})$ \\
\hline HSA NPs & $>500 \mathrm{~nm}$ & $>0.5$ & - \\
HSA NPs + Glucose & $269 \pm 3$ & $0.05 \pm 0.01$ & $-45 \pm 2$ \\
HSA NPs + Mannitol & $262 \pm 5$ & $0.06 \pm 0.01$ & $-44 \pm 1$ \\
HSA NPs + Sucrose & $261 \pm 1$ & $0.06 \pm 0.01$ & $-45 \pm 1$ \\
CS-HSA NPs & $>500 \mathrm{~nm}$ & $>0.5$ & - \\
CS-HSA NPs + Glucose & $276 \pm 5$ & $0.12 \pm 0.02$ & $+31 \pm 1$ \\
CS-HSA NPs + Mannitol & $285 \pm 2$ & $0.11 \pm 0.01$ & $+17 \pm 3$ \\
CS-HSA NPs + Sucrose & $269 \pm 3$ & $0.06 \pm 0.01$ & $+35 \pm 1$ \\
\hline
\end{tabular}

All the cryoprotectants were tested at the concentration of $2 \% \mathrm{w} / \mathrm{v}$. The best performance in terms of technological aspects was obtained with sucrose (Table 3). A slight size increase was found, nevertheless the NPs maintained physical characteristics suitable for the intranasal application.

Non-reducing compounds such as mannitol and sucrose are generally preferred to avoid potential Maillard reaction of the cryoprotectant with the protein [61]. Sucrose resulted better than mannitol in particular for CS-HSA NPs, so it was selected for storage stability studies. A possible reason for this result could be the tendency of mannitol to crystallize during the freezedrying process [61]. 


\subsection{Storage stability studies}

Physical stability of HSA NPs and CS-HSA NPs as suspension and as freeze-dried product was monitored during three months. Both HSA NPs and CS-HSA NPs suspensions resulted stable at $4^{\circ} \mathrm{C}$. The final values of particle size and PdI for HSA NPs were $238 \pm 5$ and $0.10 \pm 0.01$, respectively, while for CS-HSA NPs they resulted $265 \pm 2$ and $0.11 \pm 0.01$, respectively. No significant changes were also found for $\zeta$-potential after 3 months of storage: $-47 \pm 1$ for HSA NPs and $+43 \pm 2$ for CS-HSA NPs.

A similar study was performed on freeze-dried NPs. Both products maintained almost the same initial physical characteristics: size $260 \pm 3 \mathrm{~nm}, \operatorname{PdI} 0.07 \pm 0.01$ and $\zeta$-potential of $-40 \pm 1 \mathrm{mV}$, in the case of HSA NPs; and size $265 \pm 1 \mathrm{~nm}$, PdI $0.06 \pm 0.01$ and $\zeta$-potential of $+37 \pm 2 \mathrm{mV}$, in the case of CS-HSA NPs. These data demonstrate that the developed NPs can be stored as suspension in the fridge and potentially even at room temperature as freeze-dried products for long periods.

Stability of SulfB loaded NPs was also considered. They were physically and chemically stable at $37^{\circ} \mathrm{C}$ and in the light for three days. These results were also important for the subsequent in vitro and ex vivo experiments.

\subsection{Mucoadhesion properties}

Turbidity analyses and the evaluation of the $\zeta$-potential were synergistically applied to investigate and compare the mucoadhesion properties of CS-HSA NPs and uncoated NPs.

As clearly reported in Figure 3, a significant increase in absorbance difference $(\Delta \mathrm{A})$ was found in the case of CS-HSA NPs indicating a strong interaction between positively charged amino groups present in CS and the negatively charged carboxyl and sulphate groups of the mucin $[36,62]$. $\zeta$-potential analyses confirmed the data obtained by the turbidimetric method. Positive charges of CS-HSA NPs were neutralized by the negative charges of mucin (Figure 4). On the other hand, uncoated NPs remain negatively charged once the interaction with mucin occurs at lower extension $[36,63]$.

\subsection{In vitro release experiments}

In vitro release profiles of the dye from the aqueous solution, the SulfB-HSA NPs and the SulfB-CS-HSA NPs suspensions are reported in Figure 5. In the case of the solution, $100 \%$ of SulfB was released after $2 \mathrm{~h}$, while in the case of both formulations the immediate release of the entrapped molecule, known as burst effect, was very low. This represents an important 
finding because it suggests that the drug leakage during in vivo delivery would be minimal. The release of SulfB from CS-HSA NPs started quite slower respect to HSA NPs, then, after about four hours, the release profiles of the formulations became similar as a consequence of CS hydration and swelling (Figure 5) [51,64].

As previously reported, the release kinetics of SulfB, employed as a model of a hydrophilic drug, were analysed by Hixson-Crowell, Higuchi, first- and zero-order mathematical models. The results revealed that the release of SulfB from both formulations was diffusion-controlled, as indicated by higher R-squared $\left(\mathrm{R}^{2}\right)$ values in the Higuchi model $(0.839$ for HSA NPs and 0.884 for CS-HSA NPs).

When the release data were analyzed using the Korsemeyer-Peppas model, the values of the diffusional exponent $\mathrm{n}$ were found to be 0.68 and 0.67 for HSA NPs and CS-HSA NPs, respectively, demonstrating that the SulfB release follows a non-Fickian diffusion mechanism.

\subsection{Caco-2 experiments}

Caco-2 cell line was used as model of the nasal epithelium [23,30]. The cells used for the test are rich in mucin content, an important feature also to evaluate the effect of chitosan coating on the permeation.

After Alcian-blue PAS reaction, Caco-2 cells appeared stained in magenta, highlighting the presence of neutral mucins (Figure 6). This kind of mucins usually reacts with PAS. The lack of blue staining highlighted the absence of acid mucins [65].

A first set of experiments was performed to select the suitable dilution of NPs dispersions for further studies, i.e., permeation and uptake experiments. For this purpose, cell viability (MTS) assay was carried out. As reported in Figure 7 (panel a), when Caco-2 cells were exposed for 4 $\mathrm{h}$ to different concentrations of SulfB-HSA NPs and SulfB-CS-HSA NPs (dilution from 1:40 to 1:10) the cell viability was not reduced. Further experiments demonstrated that when the cells were incubated with the two types of SulfB-NPs for $24 \mathrm{~h}$, diluted 10 times, the cell viability was not significantly affected compared to the control group (Figure 7, panel b).

Since the developed formulations are designed for nasal administration, and the nasal epithelium represents the first barrier, these results demonstrate their biocompatibility, independently of the surface charge.

The permeation studies across the Caco-2 monolayer revealed that the $\mathrm{P}_{\text {app }}$ values of SulfB-HSA NPs and SulfB-CS-HSA NPs were similar for the first 3 hours (Figure 8). This is probably related to the mucoadhesive characteristics that CS conveys to the NPs. A delayed drug release occurs in the presence of CS-coating $[56,66,67]$. However, after $4 \mathrm{~h}$ of incubation, the $\mathrm{P}_{\text {app }}$ value 
of SulfB-CS-HSA NPs was higher, about twice the $\mathrm{P}_{\text {app }}$ value of SulfB-HSA NPs (Figure 8). Finally, the obtained $\mathrm{P}_{\text {app }}$ results are useful for in vitro prediction, as confirmed by the recovery values, which were above $80 \%$ in all experiments [27].

To elucidate the endocytic uptake mechanism and to differentiate SulfB-HSA NPs and SulfBCS-HSA NPs, the uptake experiments were carried out in the presence of sodium azide as energy depletion agent, chlorpromazine, a clathrin-dependent endocytosis inhibitor and indomethacin, as caveolin-dependent endocytosis inhibitor. As evidenced in Figure 9, the cellular uptake of both NPs was significantly inhibited in the presence of sodium azide, chlorpromazine and indomethacin with a more pronounced effect of all the inhibitors in the case of uncoated NPs. These data indicated that both formulations, were internalized by energydependent mechanism and the clathrin-mediated endocytosis resulted the main pathway involved in the uptake, consistent with the results obtained in other works with similar formulations [68].

\section{8 hCMEC/D3 experiments}

The possible toxic effects of the NPS were evaluated by MTT and LDH assays in hCMEC/D3 cell line (Figure 10). The same cells were used for permeability studies. When cells were exposed to HSA NPs and CS-HSA NPs (from dilution 1:500, 1:100 and 1:10) for 2 and $24 \mathrm{~h}$, no significant changes were observed in MTT metabolism, or LDH release respected to cells incubated with only complete medium indicating that HSA NPs and CS-HSA NPs did not affect the metabolic activity of the cells nor the membrane integrity.

A lot of study use the hCMEC/D3 cell line as a model of the human BBB and in particular for to understand the drug transport mechanisms $[15,27]$. As the cells retain the expression of most transporters and receptors expressed in vivo, the permeability studies were performed to predict the permeability of SulfB-HSA NPs and SulfB-CS-HSA NPs across the BBB, after a possible absorption via the respiratory epithelium and achievement of the circulation, as indirect pathway for the NTB delivery. NaF was used as negative control and its $\mathrm{P}_{\text {app }}$ was calculated to monitor the integrity of the cell layer, together with the phase-contrast microscopy [28].

The permeation studies across hCMEC/D3 monolayer revealed that the $\mathrm{P}_{\text {app }}$ value of SulfB-CSHSA NPs was higher than with SulfB-HSA NPs at 1 and 2 hours (Figure 11), this effect perhaps is in part due to the mechanism of action of CS which induces a reversible transient change of the tight junctions. However, after $3 \mathrm{~h}$ of incubation, the $\mathrm{P}_{\text {app }}$ values of SulfB-CS-HSA NPs and SulfB-HSA NPs were similar. 
To understand the possible endocytic uptake mechanism of the two different formulations, the sodium azide, as energy depletion agent, the chlorpromazine, a clathrin-dependent endocytosis inhibitor and the indomethacin, as caveolin-dependent endocytosis inhibitor, were used. As evidenced in Figure 12, the cellular uptake of SulfB-HSA NPs and SulfB-CS-HSA NPs was significantly reduced in the presence of all three inhibitors with a greater effect in the case of uncoated NPs. These data indicate that both formulations were internalized in an energydependent manner and the clathrin-mediated endocytosis was the main pathway involved in this uptake.

\subsection{Effects of nanoparticles on tight and gap junctions expression}

Chitosan induced a significant decrease of the expression of gap junctions in hCMEC/D3 cells, but not in tight junctions in Caco-2 (data not shown). Exposure of the two cell lines to CS-HSA NPs at the 1:10 dilution for $2 \mathrm{~h}$ caused a decrease in the levels of ZO-1 expression significantly for hCMEC/D3, but not for Caco-2. However, HSA NPs did not alter the level of the protein (Fig. 13). In the hCMEC/D3 cells CS-HSA NPs decreased also the levels of Caudin-5 and VeCadherin that are the most abundant gap junctions in these cells [27] (Fig.13). The different effect observed with the NPs in the two cell lines can depend on the different mechanisms of action of chitosan: its effect on the gap junctions in the case of hCMEC/D3 cells, while the mucoadhesion in the case of Caco-2.

Variations of tight junctions (TJP1) expression were assessed also at RNA level, by RT-PCR. As shown in Figure 14 (panel a), the exposure to CS-HSA NPs and, at a lesser extent, to HSA NPs for 2 hours, significantly reduced the expression of ZO1 in hCMEC/D3 cells while these formulations did not significantly alter its expression in Caco-2 cells, confirming the findings obtained at the protein level (Fig. 13). The qualitative PCR confirmed the data obtained and shown in real time PCR (Fig. 14, panel b).

Overall, these data indicate that CS-HSA NPs have advantages in opening the tight junctions between hCMEC/D3 cells, a model of blood-brain barrier, allowing for the transport of molecules across the barrier. The effect on Caco- 2 cells, on the contrary, indicated that these formulations can be safely used for nasal administration because intact epithelial tight junctions are crucial for maintaining barrier function. Mucoadhesion is the main mechanism at this level. 


\subsection{Ex vivo transport experiments across rabbit nasal mucosa}

The ex vivo permeation of SulfB from SulfB-HSA NPs, SulfB-CS-HSA NPs and aqueous solution was studied by using rabbit nasal mucosa as biological barrier [42-44]. The maximum permeation of SulfB in $24 \mathrm{~h}$ was $83.7 \%$ for CS-HSA NPs, $68.9 \%$ for HSA NPs and $45.5 \%$ in the case of the aqueous solution of the dye (Figure 13). The higher permeation of SulfB, when loaded into CS-HSA NPs could be due to the permeation enhancing activity of CS, in particular to the interaction of positively charged amino groups of CS with negatively charged cell membranes and to the opening of the tight junctions, as evidenced in the experiments with Caco-2 and hCMEC/D3 cells. On the other hand, the possible reason for the lower permeation of the free-SulfB in comparison to SulfB loaded into HSA NPs and CS-HSA NPs could be due to the hydrophilic nature of drug [49].

Besides, the apparent permeability coefficient of SulfB loaded into CS-HSA NPs resulted 16.7 $\pm 1.3 \times 10^{-6} \mathrm{~cm} / \mathrm{s}$, while the permeation rates of HSA NPs and free-SulfB resulted $13.8 \pm 1.1 \mathrm{x}$ $10^{-6} \mathrm{~cm} / \mathrm{s}$ and $9.1 \pm 0.1 \times 10^{-6} \mathrm{~cm} / \mathrm{s}$, respectively. A greater permeability coefficient is expected to correlate with enhanced absorption [69]. The obtained data are suitable for an accurate prediction since the mass balance values were $98.9 \pm 1.6 \%$ for SulfB-HSA NPs and $99.6 \pm 0.6$ $\%$ for SulfB-CS-HSA NPs.

The CS coating on the surface of HSA NPs determined a slower amount of SulfB permeated in particular during the first 6 hours, in comparison to uncoated NPs (Figure 15). This result is due to the mucoadhesive properties of CS-HSA NPs, which could result in an extended time of action, in accordance with the results obtained by the in vitro release studies and Caco-2 experiments [51,56,64,66,67].

The mucoadhesive properties of SulfB-CS-HSA NPs compared to SulfB-HSA NPs were also evidenced by the experiment performed for $4 \mathrm{~h}$. As hypothesized, concerning the amount of SulfB recovered into the rabbit nasal mucosa after $4 \mathrm{~h}, \mathrm{CS}-\mathrm{HSA}$ NPs exhibited higher SulfB accumulation $(6.7 \%)$ compared to HSA NPs $(1.7 \%)$. These results demonstrated the strong interaction between CS and the mucin.

\section{Conclusion}

In this study, the influence of CS-coating on HSA NPs for nasal delivery was investigated for the first time. Concerning physical and chemical parameters, the use of chitosan-coated nanoparticles is a promising approach for nose-to-brain drug delivery. The freeze-drying process was optimized, and both NPs showed excellent storage stability properties as 
suspensions, at $4^{\circ} \mathrm{C}$, and as freeze-dried products. The functionalization coating with CS was confirmed by the difference in the $\zeta$-potential value and also by TEM analysis. Besides, the mucoadhesive properties of CS and its ability to modulate the release of the entrapped molecule were proved by the turbidimetric and the indirect method and performing in vitro release studies. Caco-2 and hCMEC/D3 cell experiments, selected as model of nasal epithelium and $\mathrm{BBB}$, respectively, evidenced the safety of the NPs and the ability of CS to increase the drug permeation. Both formulations were internalized into the cells with the energy-dependent mechanism, and the clathrin-mediated endocytosis was found to be the main pathway involved in this process.

The possible effects of the two formulations on tight and gap junctions expression were assessed in both cellular lines. CS-HSA NPs have the advantage of opening the tight junctions between hCMEC/D3 cells, by decreasing the levels of ZO-1 expression, allowing for the transport of molecules across the barrier. The effect was not evidenced in the Caco-2 cells. The different effect observed with the NPs in the two cell lines may be dependent on the different mechanisms of action of $\mathrm{CS}$, in the case of hCMEC/D3 cells dependent on the gap junctions while in the case of Caco-2 on the mucoadhesion.

Ex-vivo studies using rabbit nasal mucosa demonstrated a strong interaction between CS and the mucosa and the higher penetrating potential of CS-HSA NPs respect to uncoated NPs. Consequently, the present research proposed a novel and promising strategy based on HSA NPs surface modification for the NTB delivery of many bioactives.

\section{Funding}

This research did not receive any specific grant from funding agencies in the public, commercial, or not-for-profit sectors.

\section{Acknowledgements}

TEM analyses were performed thanks to the collaboration with Dr. Maria Cristina Salvatici, Electron Microscopy Centre (Ce.M.E.), ICCOM, CNR, Sesto Fiorentino, Florence, Italy. 


\section{REFERENCES}

[1] A. Mistry, S. Stolnik, L. Illum, Nanoparticles for direct nose-to-brain delivery of drugs, Int. J. Pharm. 379 (2009) 146-157, https://doi:10.1016/j.ijpharm.2009.06.019

[2] Y. Chen, L. Liu, Modern methods for delivery of drugs across the blood-brain barrier, Adv. Drug Deliv. Rev. 64 (2012) 640-665, https://doi:10.1016/j.addr.2011.11.010

[3] A. Pires, A. Fortuna, G. Alves, A. Falcao, Intranasal Drug Delivery: How, Why and What for? J. Pharm. Pharm. Sci. 12 (2009) 288-311. https://doi:10.18433/J3nc79

[4] F. Sonvico, A. Clementino, F. Buttini, G. Colombo, S. Pescina, S.S. Guterres, A.R. Pohlmann, S. Nicoli, Surface-Modified Nanocarriers for Nose-to-Brain Delivery: From $\begin{array}{llllll}\text { Bioadhesion to } & \text { Targeting, } & \text { Pharmaceutics } & 10 & \text { (2018) }\end{array}$ https://doi.org/10.3390/pharmaceutics10010034

[5] A. Bonaccorso, T. Musumeci, M.F. Serapide, R. Pellitteri, I. F. Uchegbu, G. Puglisi, Nose to brain delivery in rats: Effect of surface charge of rhodamine B labeled nanocarriers on brain subregion localization, Colloids Surf. B Biointerfaces 154, (2017) 297-306, https://doi:10.1016/j.colsurfb.2017.03.035

[6] Y.H. Feng, H.S. He, F.Q. Li, Y. Lu, J.P. Qi, W. Wu, An update on the role of nanovehicles in nose-to-brain drug delivery. Drug Discov. Today 23 (2018) 1079-1088, https://doi:10.1016/j.drudis.2018.01.005

[7] M. Agrawal, S. Saraf, S. Saraf, S.G. Antimisiaris, M.B. Chougule, S.A. Shoyele, A. Alexander, Nose-to-brain drug delivery: An update on clinical challenges and progress towards approval of anti-Alzheimer drugs, J. Control. Release 281 (2018) 139-177, https://doi:10.1016/j.jconrel.2018.05.011

[6] Y.H. Feng, H.S. He, F.Q. Li, Y. Lu, J.P. Qi, W. Wu, An update on the role of nanovehicles in nose-to-brain drug delivery, Drug Discov. Today 23(5), (2018). 1079-1088. https://doi:10.1016/j.drudis.2018.01.005

[8] S. Md, S.K. Bhattmisra, F. Zeeshan, N. Shahzad, M.A. Mujtaba, V.S. Meka, J. Ali, Nanocarrier enabled drug delivery systems for nose to brain targeting for the treatment of neurodegenerative disorders, J. Drug Deliv. Sci. Tec. 43 (2018) 295-310, https://doi:10.1016/j.jddst.2017.09.022

[9] E. Samaridou, M.J. Alonso, Nose-to-brain peptide delivery - The potential of nanotechnology, Bioorg. Med. Chem. $26 \quad$ (2018) 2888-2905, https://doi:10.1016/j.bmc.2017.11.001

[10] A.O. Elzoghby, W.M. Samy, N.A. Elgindy, Albumin-based nanoparticles as potential controlled release drug delivery systems, J. Control. Release 157 (2012) 168-182, https://doi:10.1016/j.jconrel.2011.07.031

[11] M.C. Bergonzi, C. Guccione, C. Grossi, V. Piazzini, A. Torracchi, I. Luccarini, F. Casamenti, A.R. Bilia, Albumin Nanoparticles for Brain Delivery: A Comparison of Chemical 
versus Thermal Methods and in vivo Behavior, ChemMedChem 11 (2016) 1840-1849, https://doi:10.1002/cmdc.201600080

[12] Y.L. Tan, H.K. Ho, Navigating albumin-based nanoparticles through various drug delivery routes, Drug Discov. Today 23 (2018) 1108-1114, https://doi:10.1016/j.drudis.2018.01.051

[13] A. Jithan, K. Madhavi, M. Madhavi, K. Prabhakar, Preparation and characterization of albumin nanoparticles encapsulating curcumin intended for the treatment of breast cancer, Int. J. Pharm. Investig. 1 (2011) 119-125. https://doi:10.4103/2230-973X.82432

[14] B. Wilson, Y. Lavanya, S.R.B. Priyadarshini, M. Ramasamy, J. Leno Jenita, Albumin nanoparticles for the delivery of gabapentin: Preparation, characterization and pharmacodynamic studies, Int. J. Pharm. $473 \quad$ (2014) 73-79, http://dx.doi.org/10.1016/j.ijpharm.2014.05.056

[15] C. Guccione, M. Oufir, V. Piazzini, D. E. Eigenmann, E. A. Jahne, V. Zabela, MT. Faleschini, M.C. Bergonzi, M. Smiesko, M. Hamburger, A.R. Bilia, Andrographolide-loaded nanoparticles for brain delivery: Formulation, characterisation and in vitro permeability using hCMEC/D3 cell line, Eur. J. Pharm. Biopharm. 120 (2017) 146-146, http://doi:10.1016/j.ejpb.2017.09.010

[16] J. B. Ferrado, A.A. Perez, F.F. Visentini, G.A. Islan, G.R. Castro, L.G. Santiago, Formation and characterization of self-assembled bovine serum albumin nanoparticles as chrysin delivery systems, Colloids Surf. B Biointerfaces 173 (2018) 43-51, http://doi:10.1016/j.colsurfb.2018.09.046

[17] M.M. Elgohary, M.W. Helmy, S.M. Mortada, A.O. Elzoghby, Dual-targeted nano-in-nano albumin carriers enhance the efficacy of combined chemo/herbal therapy of lung cancer, Nanomedicine (Lond) 13 (2018) 2221-2224, http://doi:10.2217/nnm-2018-0097

[18] F. Kratz, Albumin as a drug carrier: Design of prodrugs, drug conjugates and nanoparticles, J. Control. Release 132, (2008) 171-183, https://doi.org/10.1016/j.jconrel.2008.05.010

[19] B. Luppi, F. Bigucci, G. Corace, A. Delucca, T. Cerchiara, M. Sorrenti, V.Zecchi, Albumin nanoparticles carrying cyclodextrins for nasal delivery of the anti-Alzheimer drug tacrine, Eur. J. Pharm. Sci. 44 (2011) 559-565, http://doi:10.1016/j.ejps.2011.10.002

[20] L.R. Wong, P.C. Ho, Role of serum albumin as a nanoparticulate carrier for nose-to-brain delivery of R-flurbiprofen: implications for the treatment of Alzheimer's disease, J. Pharm. Pharmacol. 70 (2018) 59-69, http://doi:10.1111/jphp.12836

[21] M. Esfandyari-Manesh, A. Mohammadi, F. Atyabi, S.M. Nabavi, S.M. Ebrahimi, E. Shahmoradi, Varnamkhasti BS, M.H. Ghahremani, R. Dinarvand, Specific targeting delivery to MUC1 overexpressing tumors by albumin-chitosan nanoparticles conjugated to DNA aptamer, Int. J. Pharm. 515 (2016) 607-615, http://doi:10.1016/j.ijpharm.2016.10.066

[22] L. Casettari, L. Illum, Chitosan in nasal delivery systems for therapeutic drugs. J. Control. Release 190 (2014) 189-200, http://doi:10.1016/j.jconrel.2014.05.003 
[23] G. Rassu, E. Soddu, M. Cossu, A. Brundu, G. Cerri, N. Marchetti, L. Ferraro, R.F. Regan, P. Giunchedi, E. Gavini, A. Dalpiaz, Solid microparticles based on chitosan or methyl-betacyclodextrin: a first formulative approach to increase the nose-to-brain transport of deferoxamine mesylate, J. Control. Release 201 (2015) 68-77, http://doi:10.1016/j.jconrel.2015.01.025

[24] Y.R. Li, H.L. Song, S.J. Xiong, T. Tian, T.B. Liu, Y.Y. Sun, Chitosan-stablized bovine serum albumin nanoparticles having ability to control the release of NELL-1 protein, Int. J. Biol. Macromol. 109 (2018) 672-680, http://doi:10.1016/j.ijbiomac.2017.12.104

[25] M. Gaber, M.T. Mabrouk, M.S. Freag, S.K. Khiste, J.Y. Fang, K.A. Elkhodairy, A.O. Elzoghby, Protein-polysaccharide nanohybrids: Hybridization techniques and drug delivery applications. Eur J Pharm Biopharm. 133 (2018) 42-62, http://doi:10.1016/j.ejpb.2018.10.001

[26] Quantification and Brain Targeting of Eugenol-Loaded Surface Modified Nanoparticles Through Intranasal Route in the Treatment of Cerebral Ischemia. Niyaz Ahmad, Rizwan Ahmad, Md Aftab Alam, Farhan Jalees Ahmad Drug Res (Stuttg) 2018; 68(10): 584-595 DOI: $10.1055 / \mathrm{a}-0596-7288$

[27] D.E. Eigenmann, G. Xue, K.S. Kim, A.V. Moses, M. Hamburger, M. Oufir, Comparative study of four immortalized human brain capillary endothelial cell lines, hCMEC/D3, hBMEC, TY10, and BB19, and optimization of culture conditions, for an in vitro blood-brain barrier model for drug permeability studies, Fluids Barriers CNS 10 (2013) 33, http://doi:10.1186/2045-8118-10-33

[28] G. Graverini, V. Piazzini, E. Landucci, D. Pantano, P. Nardiello, F. Casamenti, D. Pantano, D.E. Pellegrini-Giampietro, A.R. Bilia, M.C. Bergonzi, Solid lipid nanoparticles for delivery of andrographolide across the blood-brain barrier: in vitro and in vivo evaluation, Colloids Surf. B Biointerfaces 161 (2018) 302-313, http://doi:10.1016/j.colsurfb.2017.10.062

[29] V. Piazzini, E. Landucci, G. Graverini, D.E. Pellegrini-Giampietro, A.R. Bilia, M.C. Bergonzi, Stealth and cationic nanoliposomes as drug delivery systems to increase andrographolide BBB permeability, Pharmaceutics 2018, 10, 128, http://doi:10.3390/pharmaceutics10030128

[30] G. Rassu, E. Soddu, A.M. Posadino, G. Pintus, B. Sarmento, P. Giunchedi, E. Gavini, Nose-to-brain delivery of BACE1 siRNA loaded in solid lipid nanoparticles for Alzheimer's therapy. Colloids Surf. B Biointerfaces 152 (2017) 296-301. http://doi:10.1016/j.colsurfb.2017.01.031

[31] C. Weber, C. Coester, J. Kreuter, K. Langer, Desolvation process and surface characterisation of protein nanoparticles, Int. J. Pharm. 194 (2000) 91-102, https://doi.org/10.1016/S0378-5173(99)00370-1

[32] K. Langer, S. Balthasar, V. Vogel, N. Dinauer, H. von Briesen, D. Schubert, Optimization of the preparation process for human serum albumin (HSA) nanoparticles. Int. J. Pharm. 257, (2003) 169-180, https://doi.org/10.1016/S0378-5173(03)00134-0 
[33] M. Merodio, A. Arnedo, M.J. Renedo, J.M. Irache, Ganciclovir-loaded albumin nanoparticles: characterization and in vitro release properties. Eur. J. Pharm. Sci. 12 (2001) 251-259, https://doi.org/10.1016/S0928-0987(00)00169-X

[34] M.G. Anhorn, H.C. Mahler, K. Langer, Freeze drying of human serum albumin (HSA) nanoparticles with different excipients, Int. J. Pharm. 363 (2008) 162-169. https://doi:10.1016/j.ijpharm.2008.07.004

[35] M.C. Bonferoni, G. Sandri, F. Ferrari, S. Rossi, V. Larghi, Y. Zambito, C. Caramella, Comparison of different in vitro and ex vivo methods to evaluate mucoadhesion of glycolpalmitoyl chitosan micelles, J. Drug Deliv. Sci. Tec. 20 (2010) 419-424, https://doi:10.1016/S1773-2247(10)50073-X

[36] A.C.C. Vieira, L.L. Chaves, S. Pinheiro, S. Pinto, M. Pinheiro, S.C. Lima,. . Reis, S. (2018). Mucoadhesive chitosan-coated solid lipid nanoparticles for better management of tuberculosis. Int. J. Pharm. 536 478-485, https://doi:10.1016/j.ijpharm.2017.11.071

[37] V. Piazzini, L. Cinci, M. D’Ambrosio, C. Luceri, A.R. Bilia, M.C. Bergonzi, SLNs and Chitosan-coated SLNs as promising tool for silybin delivery: formulation, characterization, and in vitro evaluation Curr. Drug Deliv. 16 (2019) 142-152, https://doi:10.2174/1567201815666181008153602

[38] E. Bigagli, L. Cinci, M. D'Ambrosio, C. Luceri, Pharmacological activities of an eye drop containing Matricaria chamomilla and Euphrasia officinalis extracts in UVB-induced oxidative stress and inflammation of human corneal cells. J. Photochem. Photobiol. B, 173 (2017) 618625, https://doi:10.1016/j.jphotobiol.2017.06.031

[39] G. Iacomino, O. Fierro, S. D'Auria, G. Picariello, P. Ferranti, C. Liguori, F. Addeo, G. Mamone, Structural Analysis and Caco-2 Cell Permeability of the Celiac-Toxic A-Gliadin Peptide 31-55, J. Agric. Food Chem. 61 (2013) 1088-1096, https://doi:10.1021/jf3045523

[40] P. Conti, A. Pinto, L. Tamborini, U. Madsen, B. Nielsen, H. Brauner-Osborne, C. De Micheli, Novel 3-Carboxy- and 3-Phosphonopyrazoline Amino Acids as Potent and Selective NMDA Receptor Antagonists: Design, Synthesis, and Pharmacological Characterization, ChemMedChem 5 (2010) 1465-1475, https://doi:10.1002/cmdc.201000184

[41] E. Landucci, R. Lattanzi, E. Gerace, T. Scartabelli, G. Balboni, L. Negri, D.E. PellegriniGiampietro, Prokineticins are neuroprotective in models of cerebral ischemia and ischemic $\begin{array}{llllll}\text { tolerance in } & 108 \quad \text { (2016) } & \text { 39-48. }\end{array}$ https://doi:10.1016/j.neuropharm.2016.04.043

[42] P. Russo, C. Sacchetti, I. Pasquali, R. Bettini, G. Massimo, P. Colombo, A.Rossi, Primary microparticles and agglomerates of morphine for nasal insufflation. J. Pharm. Sci 95 (2006) 2553-2561, https://doi:10.1002/jps.20604

[43] F. Bortolotti, A.G. Balducci, F. Sonvico, P. Russo, G. Colombo, In vitro permeation of desmopressin across rabbit nasal mucosa from liquid nasal sprays: the enhancing effect of potassium sorbate, Eur. J. Pharm. Sci. 37 (2009) 36-42, https://doi:10.1016/j.ejps.2008.12.015 
[44] A. Giuliani, A.G. Balducci, E. Zironi, G. Colombo, F. Bortolotti, L. Lorenzini, V. Galligioni, G. Pagliuca, A. Scagliarini, L. Calzà, F. Sonvico, In vivo nose-to-brain delivery of the hydrophilic antiviral ribavirin by microparticle agglomerates, Drug Deliv. 25 (2018) 376387, https://doi:10.1080/10717544.2018.1428242

[45] A.G. Balducci, L. Ferraro, F. Bortolotti, C. Nastruzzi, P. Colombo, F. Sonvico, P. Russo, G. Colombo, Antidiuretic effect of desmopressin chimera agglomerates by nasal administration in rats, Int. J. Pharm. 440 (2013) 154-160, https://doi:10.1016/j.ijpharm.2012.09.049

[46] E. Bechgaard, S. Gizurarson, L. Jørgensen, R. Larsen, The viability of isolated rabbit nasal mucosa in the Ussing chamber, and the permeability of insulin across the membrane. Int. J. Pharm. 87 (1992) 125-132, https://doi:10.1016/0378-5173(92)90235-t

[47] M.C. Schmidt, Simmen, D., Hilbe, M., Boderke, P., Ditzinger, G., Sandow, J., S. Lang, W. Rubas, H. P. MerkleValidation of Excised Bovine Nasal Mucosa as In Vitro Model to Study Drug Transport and Metabolic Pathways in Nasal Epithelium, J. Pharm. Sci. 89 (2000) 396407, https://doi:10.1002/(sici)1520-6017(200003)89:3<396::Aid-jps10>3.0.Co;2-f

[48] P.M. Ved, K. Kim, Poly(ethylene oxide/propylene oxide) copolymer thermo-reversible gelling system for the enhancement of intranasal zidovudine delivery to the brain, Int. J. Pharm. 411 (2011) 1-9, https://doi:10.1016/j.ijpharm.2011.02.040

[49] N.K. Bari, M. Fazil, M.Q. Hassan, M.R. Haider, B. Gaba, J.K. Narang, S. Baboota, J. Ali, Brain delivery of buspirone hydrochloride chitosan nanoparticles for the treatment of general anxiety disorder, Int. J. Biol. Macromol. 81 (2015) 49-59, https://doi:10.1016/j.ijbiomac.2015.07.041

[50] A. Javia, H. Thakkar, Intranasal delivery of tapentadol hydrochloride-loaded chitosan nanoparticles: formulation, characterisation and its in vivo evaluation, J. Microencapsul. 34 (2017) 644-658, https://doi:10.1080/02652048.2017.1375038

[51] R. Raj, S. Wairkar, V. Sridhar, R. Gaud, Pramipexole dihydrochloride loaded chitosan nanoparticles for nose to brain delivery: Development, characterization and in vivo antiParkinson activity, Int. J. Biol. Macromol. $109 \quad$ (2018) 27-35, https://doi:10.1016/j.ijbiomac.2017.12.056

[52] C.C. Westcott, (1978). pH measurements. New York a.o.: Academic Press.

[53] H. Mohammad-Beigi, S.A. Shojaosadati, D. Morshedi, N. Mirzazadeh, A. Arpanaei, The Effects of Organic Solvents on the Physicochemical Properties of Human Serum Albumin Nanoparticles, Iranian J. Biotechnol. 14 (2016) 45-50, https://doi:10.15171/ijb.1168

[54] S.S. Likhodii, I. Serbanescu, M.A. Cortez, P. Murphy, O.C. Snead, W.M. Burnham, Anticonvulsant properties of acetone, a brain ketone elevated by the ketogenic diet, Ann. Neurol. 54 (2003) 219-226, https://doi:10.1002/ana.10634

[55] E. Adriaens, M.M., Dhondt, J.P. Remon, Refinement of the Slug Mucosal Irritation test as an alternative screening test for eye irritation, Toxicol. In Vitro, 19 (2005) 79-89, https://doi:10.1016/j.tiv.2004.06.004 
[56] P. Fonte, T. Nogueira, C. Gehm, D. Ferreira, B. Sarmento, Chitosan-coated solid lipid nanoparticles enhance the oral absorption of insulin, Drug Deliv. Transl. Res. 1 (2011) 299308, https://doi:10.1007/s13346-011-0023-5

[57] L. Bugnicourt, C. Ladaviere, A close collaboration of chitosan with lipid colloidal carriers for drug delivery applications, J. Control. Release 256 (2017) 121-140, https://doi:10.1016/j.jconrel.2017.04.018

[58] A.S. Tzeyung, S. Md, S.K. Bhattamisra, T. Madheswaran, N.A. Alhakamy, H.M. Aldawsari, A.K. Radhakrishnan, Fabrication, Optimization, and Evaluation of RotigotineLoaded Chitosan Nanoparticles for Nose-To-Brain Delivery. Pharmaceutics 11 (2019) 26. https://doi:10.3390/pharmaceutics11010026

[59] W. Abdelwahed, G. Degobert, S. Stainmesse, H. Fessi, Freeze-drying of nanoparticles: formulation, process and storage considerations, Adv. Drug Deliv. Rev. 58 (2006) 1688-1713, https://doi:10.1016/j.addr.2006.09.017

[60] M.K. Lee, M.Y. Kim, S. Kim, J. Lee, Cryoprotectants for freeze drying of drug nanosuspensions: effect of freezing rate, J. Pharm. Sci. 98 (2009) 4808-4817. https://doi:10.1002/jps.21786

[61] H.R. Costantino, M. J. Pikal, Lyophilization of biopharmaceuticals. (2004) Arlington, VA: AAPS Press, c2004. ISBN 0971176760

[62] Y.C. Luo, Z. Teng, Y. Li, Q. Wang, Solid lipid nanoparticles for oral drug delivery: Chitosan coating improves stability, controlled delivery, mucoadhesion and cellular uptake, Carbohydr. Polym. 122 (2015) 221-229, https://doi:10.1016/j.carbpol.2014.12.084

[63] A. Grenha, B. Seijo, C. Remunan-Lopez, Microencapsulated chitosan nanoparticles for lung protein delivery, Eur. J. Pharm. Sci. 25 (2005) 427-437, https://doi:10.1016/j.ejps.2005.04.009

[64] R. Singh, J.W. Lillard, Nanoparticle-based targeted drug delivery, Exp. Mol. Pathol. 86 (2009) 215-223, https://doi:10.1016/j.yexmp.2008.12.004

[65] A. Ferraretto, M. Bottani, P. De Luca, L. Cornaghi, F. Arnaboldi, M. Maggioni, A Fiorilli, E. Donetti, Morphofunctional properties of a differentiated Caco2/HT-29 co-culture as an in vitro model of human intestinal epithelium, Biosci. Rep. (2018) 38 BSR20171497, https://doi.org/10.1042/BSR20171497

[66] C. Prego, M. Garcia, D. Torres, M.J. Alonso, Transmucosal macromolecular drug delivery, J. Control. Release 101 (2005) 151-162, https://doi:10.1016/j.jconrel.2004.07.030

[67] F.N.S. Fachel, B. Medeiros-Neves, M. Dal Pra, R.S. Schuh, K.S. Veras, Bassani, V. L., L. Scherer Koester, A.M. Henriques, E. Braganhol, H. F. Teixeira, Box-Behnken design optimization of mucoadhesive chitosan-coated nanoemulsions for rosmarinic acid nasal delivery-In vitro studies, Carbohydr. Polym. $199 \quad$ (2018) 572-582, https://doi:10.1016/j.carbpol.2018.07.054 
[68] H.J. Byeon, Q. Thao le, S. Lee, S.Y. Min, E.S. Lee, B.S. Shin, Y.S. Youn, Doxorubicinloaded nanoparticles consisted of cationic- and mannose-modified-albumins for dual-targeting in brain tumors. J. Control. Release 225 (2016) 301-313, https://doi:10.1016/j.jconrel.2016.01.046

[69] G. Chen, D. Svirskis, W. Lu, M. Ying, Y. Huang, J. Wen, N-trimethyl chitosan nanoparticles and CSKSSDYQC peptide: N-trimethyl chitosan conjugates enhance the oral bioavailability of gemcitabine to treat breast cancer. J. Control. Release 277 (2018) 142-153. https://doi:10.1016/j.jconrel.2018.03.013 


\section{Captions to figures}

Figure 1. TEM image of HSA NPs.

\section{Figure 2. TEM image of CS-HSA NPs.}

Figure 3. Turbidimetric method. Absorbance difference of mucin admixed with HSA NPs and CSHSA NPs. Data displayed as mean $\pm \mathrm{SD} ; \mathrm{n}=3 . * * p<0.01$ vs. HSA NPs (ANOVA + Tukey's test).

Figure 4. Indirect method. $\zeta$-potential of HSA NPs and CS-HSA NPs after incubation with mucin. Data displayed as mean $\pm \mathrm{SD} ; \mathrm{n}=3$.

Figure 5. In vitro release profiles of SulfB solution, SulfB-loaded HSA NPs and SulfB-loaded CSHSA NPs in PBS. Data displayed as mean $\pm \mathrm{SD} ; \mathrm{n}=3$.

Figure 6. Mucins identification by Alcian-blue PAS staining. Left panel: representative images of Caco-2 cells x400 magnification. Right panel: detail of left panel (x1000 magnification). Scale bar: $100 \mu \mathrm{m}$.

Figure 7. Caco-2 cell viability evaluated by MTS assay after exposure for $2 \mathrm{~h}$ (a) and $24 \mathrm{~h} \mathrm{(b)}$ to HSA NPs and CS-HSA NPs. Data are expressed as percentage of control (untreated group). Values represent the mean \pm SEM of at least three experiments performed in triplicate.

Figure 8. Permeation studies across Caco- 2 cells. Data displayed as mean $\pm \mathrm{SD} ; \mathrm{n}=3$. $* * p<0.01$ vs HSA NPs. (ANOVA + Tukey's test).

Figure 9. Effect of different inhibitors on Caco-2 cell internalization pathways of HSA NPs and CSHSA NPs. Data displayed as mean $\pm \mathrm{SD} ; \mathrm{n}=3$. ${ }^{* *} p<0.01$ vs. $37^{\circ} \mathrm{C}$; \#, @, ${ }^{\circ} p<0.05$ vs. CS-HSA NPs. (ANOVA + Tukey's test).

Figure 10. hCMEC/D3 cell viability evaluated by MTT assay (right panel) and cytotoxicity by LDH assay (left panel) when exposed for $2 \mathrm{~h} \mathrm{(a),} \mathrm{and} 24 \mathrm{~h}$ (b) to HSA NPs and CS-HSA NPs. Data are expressed as percentage of control (EBM-2 medium) and Triton-X which represent, respectively, the maximum cell viability and cell cytotoxicity. Values represent the mean $\pm \mathrm{SEM}$ of at least three experiments performed in triplicate. ${ }^{* *} p<0.01 \mathrm{vs}$. EBM-2 alone.

Figure 11. Permeation studies across hCMEC/D3 cells. Data displayed as mean $\pm \mathrm{SD} ; \mathrm{n}=3$. ${ }^{*} p<$ 0.05 vs HSA NPs. (ANOVA + Tukey's test).

Figure 12. Effect of different inhibitors on hCMEC/D3 cell internalization pathways of HSA NPs and CS-HSA NPs. Data displayed as mean $\pm \mathrm{SD} ; \mathrm{n}=3 .{ }^{*} p<0.01$ vs. $37^{\circ} \mathrm{C} ;{ }^{*} p<0.05$ vs. $37^{\circ} \mathrm{C}$. (ANOVA + Tukey's test).

Figure 13. Effect of HSA NPs and CS-HSA NPs on the gap junctions in hCMEC/D3 cells. Data displayed as mean $\pm \mathrm{SD} ; \mathrm{n}=3 .{ }^{*} p<0.05$ vs. CRL. (ANOVA + Tukey's test).

Figure 14. (a) Results of the RT-PCR analyses on RNA extracted from Caco-2 and hCMEC/D3 cells exposed to HSA NPs and CS-HSA NPs, for 2 hours. Data are means \pm SE; $n=3$. ${ }^{* *} p<0.01$ and $* * * p<0.0001$ vs control (b) Representative analysis of TJP1 expression in hCMEC/D3 and CaCo-2 cells. PCR products were separated on $1.8 \%$ agarose gel containing Safeview. 
Figure 15. Ex-vivo permeation studies of SulfB solution, SulfB-HSA NPs and SulfB-CS-HSA NPs conjugates through rabbit nasal mucosa. Data displayed as mean $\pm \mathrm{SD} ; \mathrm{n}=3$. ${ }^{* *} p<0.01$ vs. SulfB solution; * $p<0.05$ vs. SulfB solution. (ANOVA + Tukey's test). 


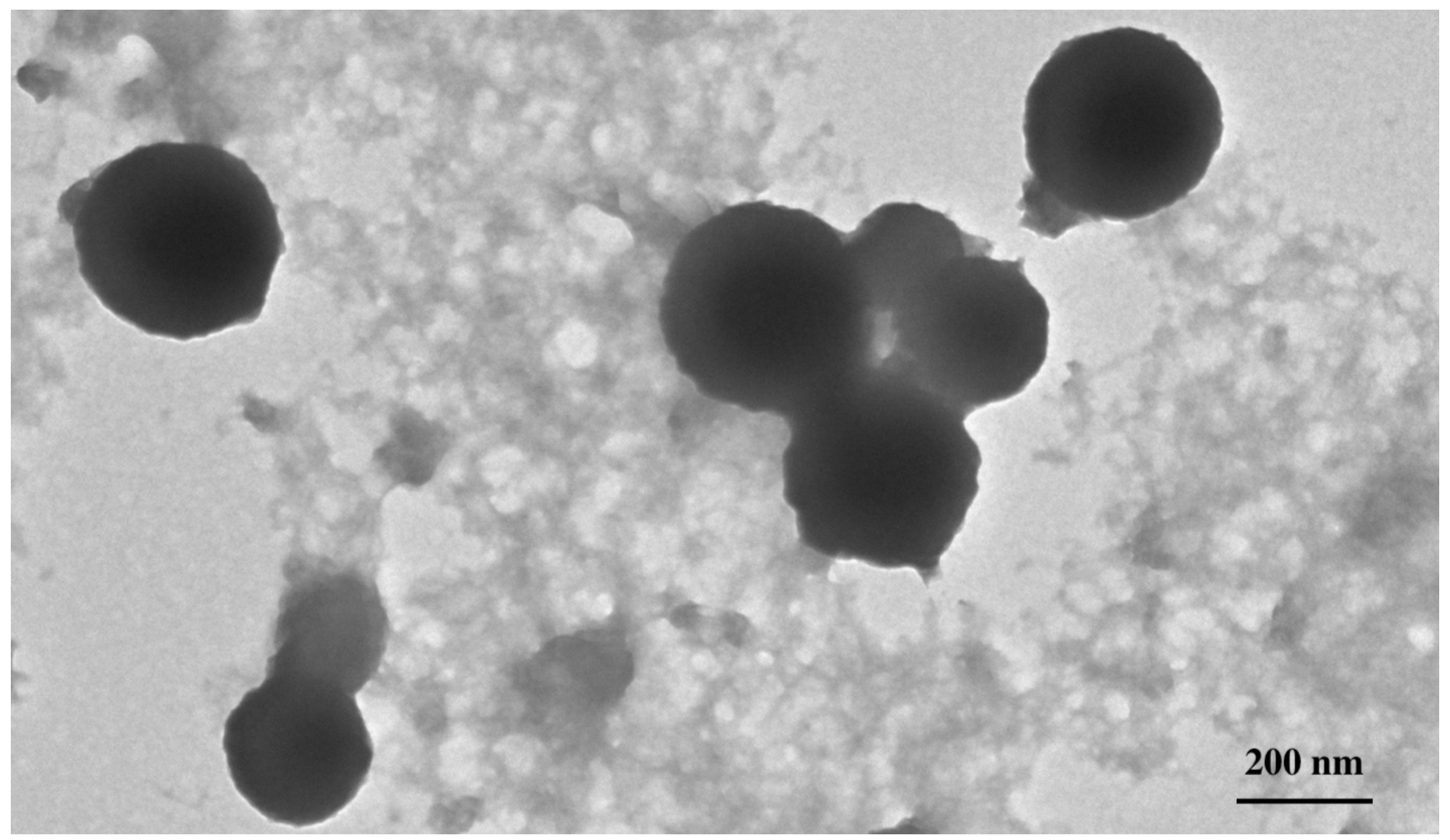




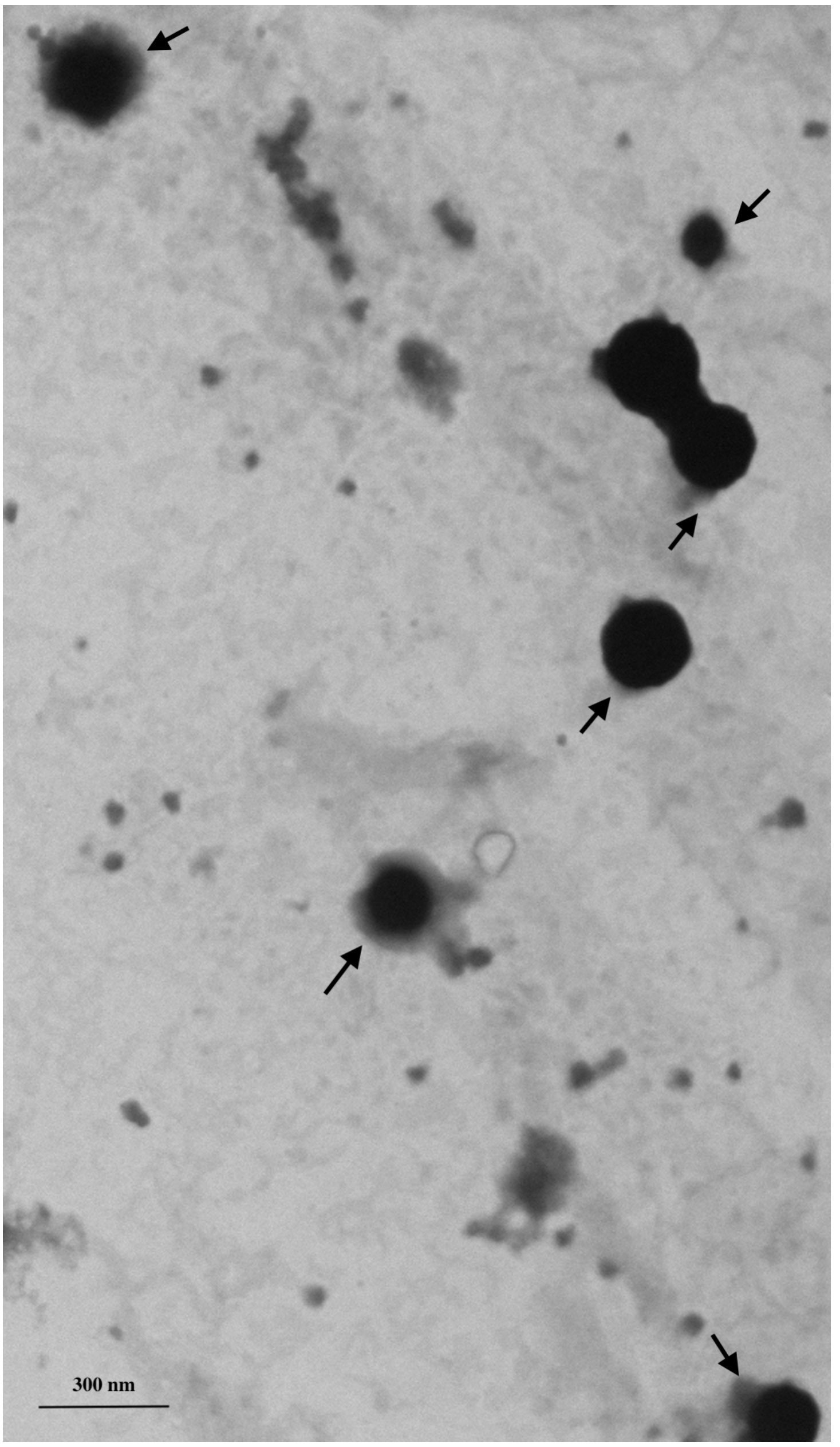




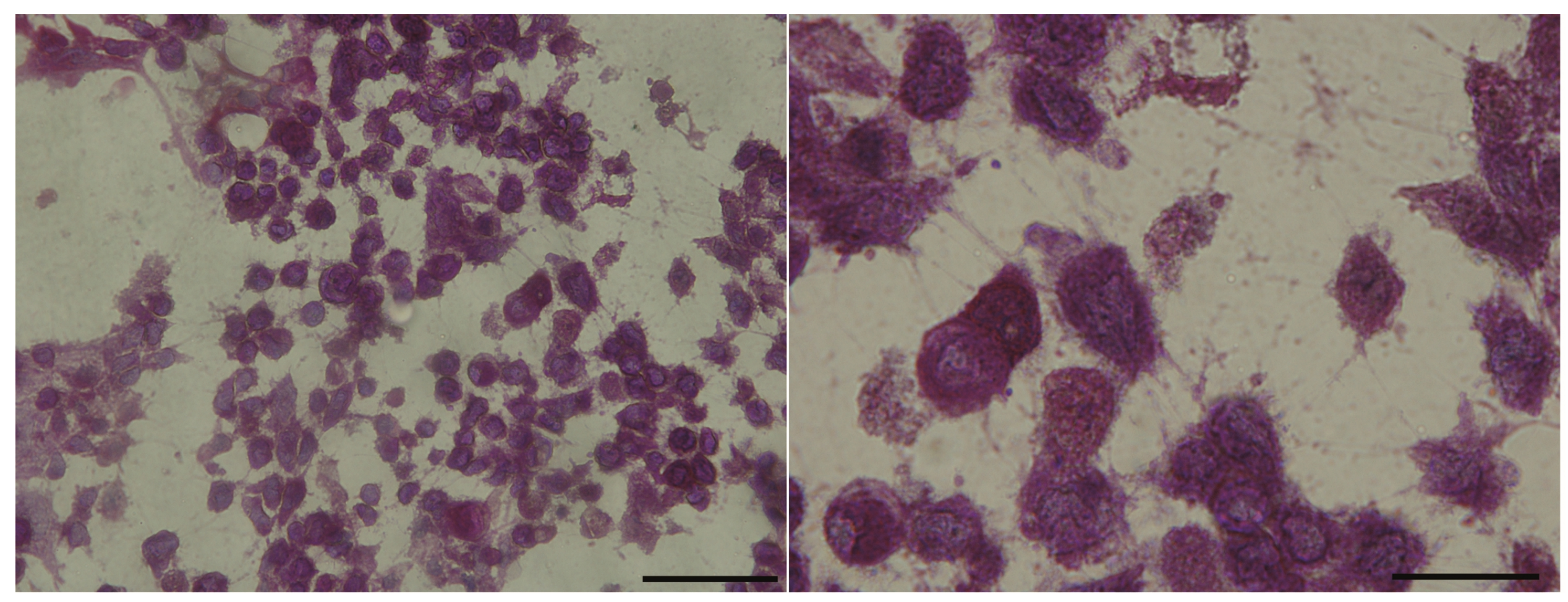



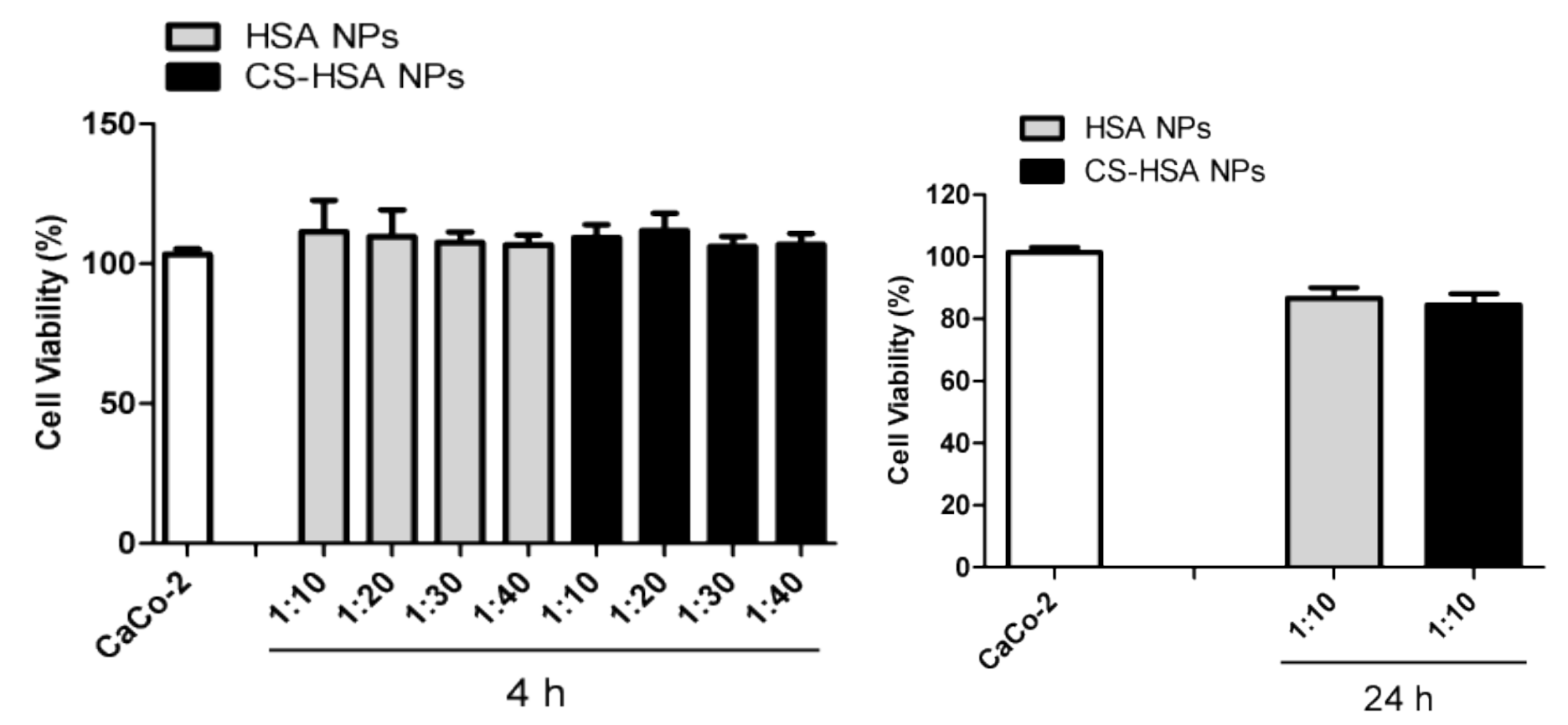


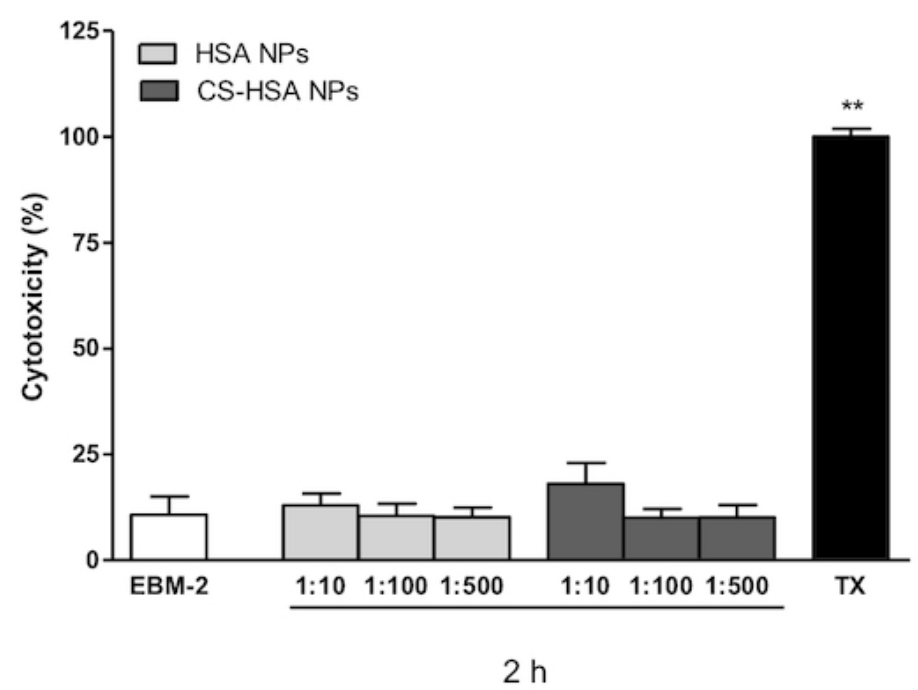

A $\square$ HSANPS

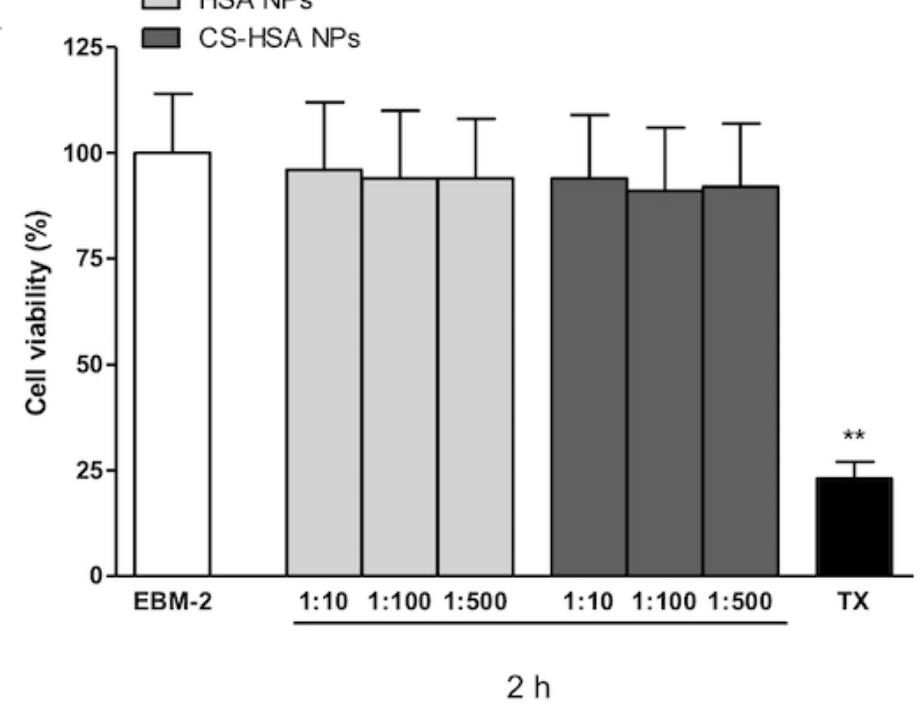

B
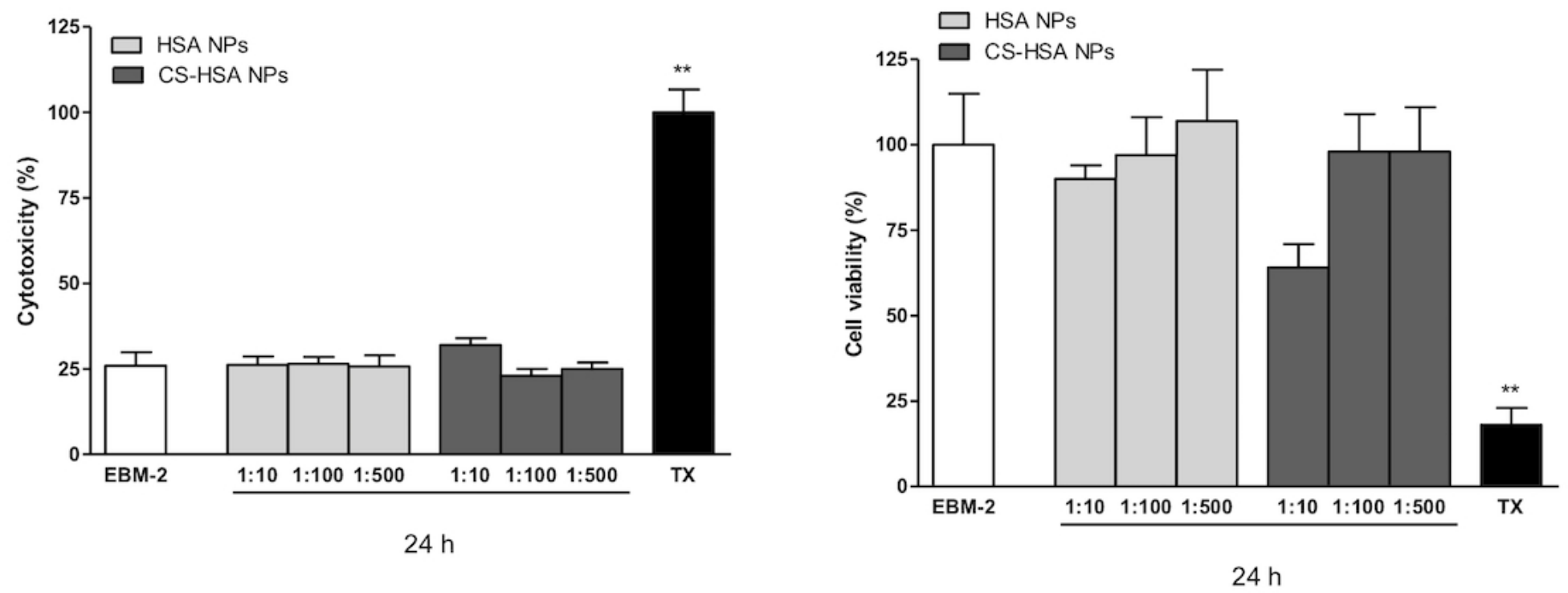

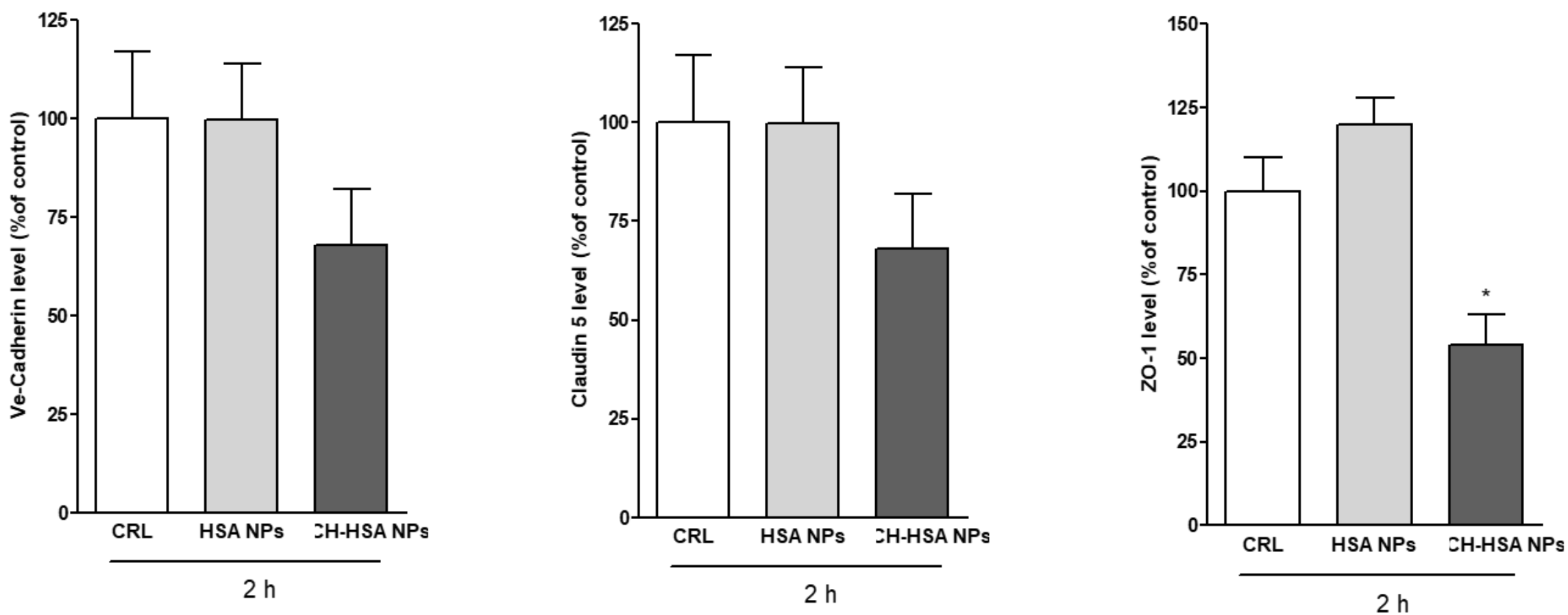
(a)

TJP1

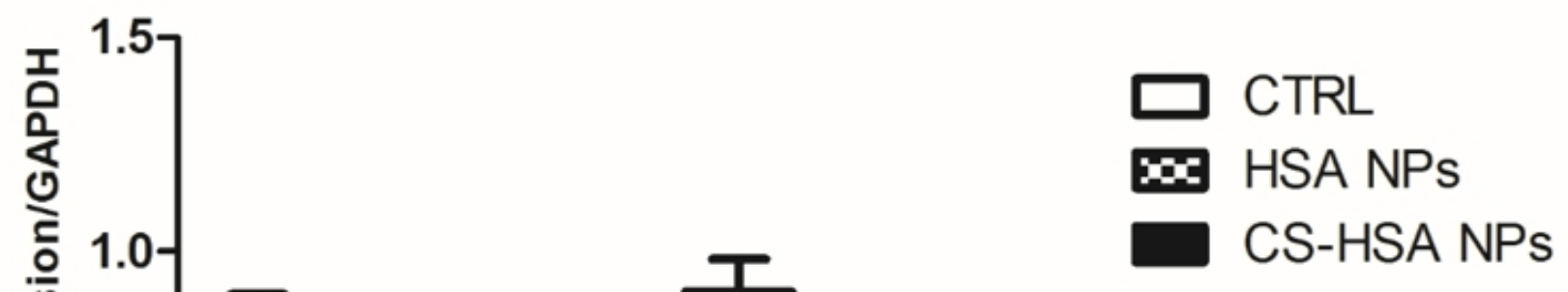

(b)

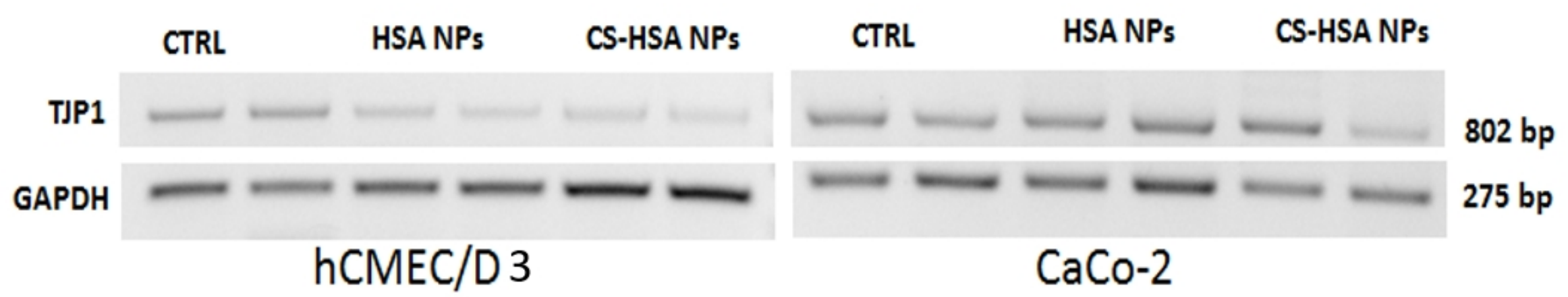

